\title{
Topological elementary equivalence of closed semi-algebraic sets in the real plane*
}

\author{
Bart Kuijpers, Jan Paredaens, University of Antwerp ${ }^{\dagger}$ \\ Jan Van den Bussche, University of Limburg ${ }^{\ddagger}$
}

\begin{abstract}
We investigate topological properties of subsets $S$ of the real plane, expressed by first-order logic sentences in the language of the reals augmented with a binary relation symbol for $S$. Two sets are called topologically elementary equivalent if they have the same such first-order topological properties. The contribution of this paper is a natural and effective characterization of topological elementary equivalence of closed semi-algebraic sets.
\end{abstract}

\section{Introduction and summary}

By viewing subsets of the real plane $\mathbf{R}^{2}$ as binary relations over the real numbers, we can use first-order logic in the language of the reals, augmented with a binary relation symbol $S$, to express properties of such sets. For example, to express that a set $S$ contains a straight line one would write the sentence

$$
(\exists a)(\exists b)(\exists c)(\neg(a=0 \wedge b=0) \wedge((\forall x)(\forall y)(S(x, y) \leftrightarrow a x+b y+c=0))),
$$

*A preliminary report of this paper was presented at the 6th International Conference on Database Theory, Delphi, Greece, January 1997.

${ }^{\dagger}$ Dept. Math. \& Computer Sci., University of Antwerp (UIA), Universiteitsplein 1, B-2610 Antwerp, Belgium. E-mail: kuijpers@uia.ua.ac.be, pareda@uia.ua.ac.be.

${ }^{\ddagger}$ Department WNI, University of Limburg (LUC), Universitaire Campus, B-3590 Diepenbeek, Belgium. E-mail: vdbuss@luc.ac.be. 
or to express that the set contains a disk as a subset, one would write

$$
\left(\exists x_{0}\right)\left(\exists y_{0}\right)(\exists r \neq 0)(\forall x)(\forall y)\left(\left(x-x_{0}\right)^{2}+\left(y-y_{0}\right)^{2}<r^{2} \rightarrow S(x, y)\right) .
$$

In this paper, we are interested in such first-order properties that are topological, in the sense that they are invariant under homeomorphisms of $\mathbf{R}^{2}$. The sentence $(\dagger)$ above gives an example of this; a semi-algebraic set contains a disk if and only if its topological interior is non-empty, and this is a purely topological property of the set. In contrast, the sentence $(*)$ is not topological.

Often, one is only interested in a certain class $\mathcal{C}$ of sets; then a property is called topological with respect to $\mathcal{C}$ if for any two sets $A$ and $B$ in $\mathcal{C}$, if $B$ is the image of $A$ under a homeomorphism of $\mathbf{R}^{2}$, then either $A$ and $B$ both satisfy the property, or neither $A$ nor $B$ do.

There is not much understanding yet of the class of those first-order sentences that are topological. One of the natural questions that arise in this respect is that of understanding topological elementary equivalence. Two sets from some class $\mathcal{C}$ are called topologically elementary equivalent (with respect to $\mathcal{C}$ ) if they satisfy precisely the same first-order properties that are topological with respect to $\mathcal{C}$.

In this paper, we focus on the class of semi-algebraic sets. A subset of $\mathbf{R}^{2}$ is called semi-algebraic if it is first-order definable in the structure of the reals as a binary relation over the reals. We moreover restrict attention to semi-algebraic sets that are closed in the ordinary topological sense.

We have been able to find a natural characterization of topological elementary equivalence of closed semi-algebraic sets in $\mathbf{R}^{2}$. Our characterization is based on a known topological property of semi-algebraic sets [6, 9], namely that locally around each point they are "conical". We partition the points in the semi-algebraic set according to the types of their cones. Roughly, our characterization then says that two closed semi-algebraic are topologically elementary equivalent if and only if the cardinalities of the equivalence classes of their partitions match.

A corollary of our characterization is that topological elementary equivalence is a decidable property of closed semi-algebraic sets. Another corollary is that topological elementary equivalence is the same as topological elementary equivalence with respect to first-order sentences in which no arithmetic, but the order predicate, is allowed. 
Our proof of the characterization involves various techniques. For the if-direction we show that there is a topological first-order sentence that expresses that the cone around a point has some specific type. For the only-if direction we show that two closed semi-algebraic sets with matching equivalence classes can be transformed into one and the same "canonical" semialgebraic set. The transformation rules used in this transformation are shown to produce topologically elementary equivalent semi-algebraic sets. The proof of the latter uses a recent collapse theorem on the expressiveness of first-order logic over the reals by Benedikt, Dong, Libkin and Wong [3], and involves reduction techniques inspired by those introduced by Grumbach and $\mathrm{Su}$ [13].

Our work is concerned with the intrinsic topological properties of single semi-algebraic sets and deals with a notion of equivalence (namely, elementary equivalence) which comes from model theory. Our work is therefore related to the large body of work in the intersection of topology and model theory (see, e.g., $[12,15,18,20]$ ). In particular, it is in the spirit of the work of Henson, Jockusch, Rubel, and Takeuti [15] who discuss a notion of elementary equivalence of topological spaces (which is also weaker than the classical notion of equivalence of topological spaces by homeomorphisms) and related invariants. These authors call two topological spaces elementary equivalent if the lattices of their closed subsets have the same first-order properties. Their notion of elementary equivalence is different from ours, however. Indeed, in their context the property of being homeomorphic to the closed unit disc is first-order expressible, while in our context there is no topological first-order sentence that distinguishes between one closed disc and two separate closed discs. In the context of Henson, Jockusch, Rubel, and Takeuti the open unit disk and the complete real plane are not distinguishable because they are homeomorphic toplogical spaces, while in our setting there clearly exists a topological first-order sentence that distinguishes between these spaces (viewed as subspaces of the real plane).

This paper is organized as follows. Definitions are given in Section 2. The partition of a closed semi-algebraic set according to the cone types of its points is described in Section 3. The main results are formulated in Section 4. The proof of the main result is given in Section 5. Two corollaries are presented in Sections 6. Concluding remarks are given in Section 7. 


\section{Preliminaries}

In this section, we give the basic definitions we will be using concerning semi-algebraic sets and topological first-order sentences.

Closed semi-algebraic sets. The standard structure $(\mathbf{R}, 0,1,+, \times,<)$ of the real numbers will be denoted simply by $\mathbf{R} .{ }^{1}$ A semi-algebraic set in $\mathbf{R}^{2}$ is a subset of $\mathbf{R}^{2}$ that is definable by a first-order formula in $\mathbf{R}$ without parameters, viewed as a binary relation over the reals. Henceforth the adjective 'in $\mathbf{R}^{2}$, will be implicitly understood and therefore omitted.

First-order logic. We will work in the language $L=(0,1,+, \times,<, S)$, being the expansion of the language of the reals with the binary relation symbol $S .^{2}$ A subset $A$ of $\mathbf{R}^{2}$ can be naturally viewed as an $L$-structure, namely the expansion $(\mathbf{R}, A)$ of $\mathbf{R}$ with $A$. Hence, the truth of an $L$-sentence $\varphi(S)$ in $(\mathbf{R}, A)$ will simply be denoted by $A \models \varphi(S)$. The sentences $(*)$ and $(\dagger)$ from the Introduction are examples of $L$-sentences. Note that, since semi-algebraic sets are first-order definable in $\mathbf{R}$, the question of $A \models \varphi(S)$, given $\varphi(S)$ and a definition of a semi-algebraic set $A$, is effectively decidable, because the first-order theory of $\mathbf{R}$ is decidable [7, 8, 19, 23].

Homeomorphism-invariance and equivalence. We call two subsets $A$ and $B$ of $\mathbf{R}^{2}$ homeomorphic if there is a homeomorphism $h$ of $\mathbf{R}^{2}$ such that $h(A)=B$. A sentence $\varphi(S)$ is called invariant under homeomorphisms (abbreviated as $\mathcal{H}$-invariant) if for any two homeomorphic semialgebraic sets $A$ and $B,(A \models \varphi(S)) \Leftrightarrow(B \models \varphi(S))$. Finally, two subsets $A$ and $B$ of $\mathbf{R}^{2}$ are called $\mathcal{H}$-equivalent if for each $\mathcal{H}$-invariant sentence $\varphi(S)$, $(A \models \varphi(S)) \Leftrightarrow(B \models \varphi(S))$.

Of course, homeomorphic semi-algebraic sets are also $\mathcal{H}$-equivalent, but the converse does not hold. For example, we will see later that if $A$ consists

\footnotetext{
${ }^{1}$ The main result of this paper remains valid if constants for all real numbers are added to the language.

${ }^{2}$ In some proofs we also use formulas in the language of the reals expanded with two additional unary relation symbols, as well as formulas in restrictions of the previous languages where $0,1,+$, and $\times$ are not used. In all these cases, similar definitions and notations are used.
} 

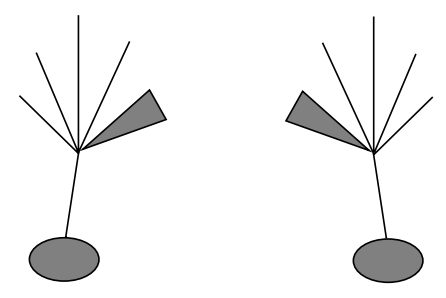

Figure 1: Two homeomorphic, but not isotopic semi-algebraic sets.

of a single closed disk, and $B$ consists of two separate closed disks, then $A$ and $B$ are $\mathcal{H}$-equivalent.

Isotopy-invariance and equivalence. It is known (e.g., $[10,16,21])$ that any orientation-preserving homeomorphism of $\mathbf{R}^{2}$ is isotopic to the identity mapping of $\mathbf{R}^{2}$. We will therefore, for reasons of convenience, refer to an orientation-preserving homeomorphism of $\mathbf{R}^{2}$ as an isotopy of $\mathbf{R}^{2}$. The prototypical example of a homeomorphism that is not an isotopy is a reflection. As a matter of fact, every homeomorphism of $\mathbf{R}^{2}$ either is an isotopy, or is isotopic to a reflection.

We call two subsets $A$ and $B$ of $\mathbf{R}^{2}$ isotopic if there is an isotopy $h$ of $\mathbf{R}^{2}$ such that $h(A)=B$. Hence, when $A$ and $B$ are homeomorphic, either $A$ is actually isotopic to $B$, or $A$ is isotopic to the mirror image of $B$. For example, Figure 1 shows two (semi-algebraic) sets that are mirror-images of each other but that are not isotopic. They can be thought of as a left hand and a right hand, where the arm and the thumb have thickness and the wrist and the other fingers have no thickness.

A sentence $\varphi(S)$ is called invariant under isotopies (abbreviated as $\mathcal{I}$ invariant) if for any two isotopic semi-algebraic sets $A$ and $B,(A \models \varphi(S)) \Leftrightarrow$ $(B \models \varphi(S))$. Finally, two subsets $A$ and $B$ of $\mathbf{R}^{2}$ are called $\mathcal{I}$-equivalent if for each $\mathcal{I}$-invariant sentence $\varphi(S),(A=\varphi(S)) \Leftrightarrow(B \models \varphi(S))$.

Of course, isotopic semi-algebraic sets are $\mathcal{I}$-equivalent, but, as mentioned above, the converse is not true. Note that $\mathcal{H}$-invariance implies $\mathcal{I}$-invariance, and that $\mathcal{I}$-equivalence implies $\mathcal{H}$-equivalence.

Examples. The sentence $(*)$ from the Introduction is a typical example of a non-topological sentence: it is neither $\mathcal{I}$-invariant nor $\mathcal{H}$-invariant. 
The sentence $(\dagger)$ from the Introduction, expressing that the topological interior is not empty, is $\mathcal{H}$-invariant (and hence also $\mathcal{I}$-invariant), and so is

$$
(\exists r)(\forall x)(\forall y)\left(S(x, y) \rightarrow x^{2}+y^{2} \leq r^{2}\right),
$$

expressing that the set is bounded, as well as

$$
\begin{aligned}
&(\exists x)(\exists y)(S(x, y) \wedge(\exists \varepsilon \neq 0) \\
&\left.\left(\forall x^{\prime}\right)\left(\forall y^{\prime}\right)\left(\left(x-x^{\prime}\right)^{2}+\left(y-y^{\prime}\right)^{2}<\varepsilon^{2} \wedge S\left(x^{\prime}, y^{\prime}\right)\right) \rightarrow\left(x^{\prime}=x \wedge y^{\prime}=y\right)\right),
\end{aligned}
$$

expressing that the set contains isolated points.

Consider a sentence expressing that for each point $p$ in the set all sufficiently small circles around $p$ intersect the set in one or two points only. Such a sentence is true in a semi-algebraic set exactly when the set consists exclusively of lines that do not intersect. This is not true for arbitrary subsets of $\mathbf{R}^{2}$; it is possible to homeomorphically distort a straight line segment so that sufficiently small circles around a certain point intersect the set infinitely often. However, since our definition of $\mathcal{H}$-invariance restricts attention to semi-algebraic sets, this sentence is $\mathcal{H}$-invariant.

Another natural topological property of sets one might want to express is topological connectivity; however, this property is not first-order, not even when restricting attention to semi-algebraic sets $[3,13]$.

\section{The point-structure of a closed semi-alge- braic set in $\mathbf{R}^{2}$}

In this section, we define the "point-structure" of a closed semi-algebraic set in $\mathbf{R}^{2}$. This definition is based on a known topological property of semialgebraic sets, namely that locally around each point they are conical $[6,9]$.

More precisely, if we denote the closed ball in $\mathbf{R}^{2}$ with center $p$ and radius $\varepsilon(\varepsilon>0)$ by $B^{2}(p, \varepsilon)$ and its bordering sphere by $S^{1}(p, \varepsilon)$, Theorem 9.3.5 of [6], specialized to $\mathbf{R}^{2}$, reads:

Property 1 Let $A$ be a semi-algebraic set in $\mathbf{R}^{2}$. For every non-isolated point $p$ of $A$ there exists an $\varepsilon>0$ and an homeomorphism $h$ of $B^{2}(p, \varepsilon)$ such that 
(i) $d(h(q), p)=d(q, p)$ for each $q \in B^{2}(p, \varepsilon),^{3}$

(ii) $h$ restricted to $S^{1}(p, \varepsilon)$ is the identity,

(iii) $h\left(A \cap B^{2}(p, \varepsilon)\right)$ is a cone with top $p$ and base $A \cap S^{1}(p, \varepsilon)$.

Also, for every isolated point $p$ of a semi-algebraic set, there exists an $\varepsilon>0$ such that $B^{2}(p, \varepsilon) \cap A=\{p\}$, so in this case we could regard it to be locally homeomorphic to a cone with an empty base.

If $A$ is a closed semi-algebraic subset of $\mathbf{R}^{2}$, the set $A \cap S^{1}(p, \varepsilon)$ from (iii) of Property 1 is a closed semi-algebraic subset of $S^{1}(p, \varepsilon)$. Since a semialgebraic set in $\mathbf{R}^{2}$ is the disjoint union of a finite number of semi-algebraic sets that are homeomorphic to the open unit disk, to the open unit interval or to a point (see Theorem 2.3.6 in [6]), $A \cap S^{1}(p, \varepsilon)$ has one of the following forms:

(1) the complete circle $S^{1}(p, \varepsilon)$,

(2) a finite number of closed arc segments and points on $S^{1}(p, \varepsilon)$, or

(3) empty.

Case (1) corresponds to interior points of $A$, Case (3) to isolated points of $A$. We use the following finite representation for these subsets of circles. A complete circle $S^{1}(p, \varepsilon)$ is represent by the letter $F$ (for "full"). For (2), we use a circular list over the alphabet $\{L, R\}$ which describes the subset of $S^{1}(p, \varepsilon)$ in a complete clockwise turn by using an $L$ for a point (this point corresponds to a "line" in the semi-algebraic set) and an $R$ for a closed arc segment (an arc segment corresponds to a "region" in the semi-algebraic set) starting from an arbitrary point outside the subset to be described. For the case of an empty set, we use the empty circular list ( ) to represent it.

Property 1 is illustrated in Figure 2. There, the cone of the point $p$ in $A$ has the representation $(L L R L R)$.

The above discussion gives rise to the following definition of "the cone" of a point in closed semi-algebraic set in $\mathbf{R}^{2}$.

\footnotetext{
${ }^{3}$ The expression $d(p, q)$ denotes the Euclidean distance between the points $p$ and $q$.
} 

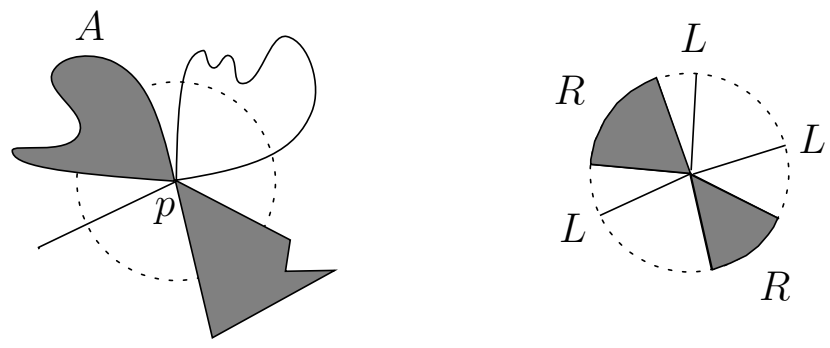

Figure 2: A closed semi-algebraic set $A$ and the cone of its points $p$ represented by the circular list $(L L R L R)$.

Definition 1 Let $A$ be a closed semi-algebraic set in $\mathbf{R}^{2}$ and let $p$ be a point of $A$. We define the cone of $p$ in $A$ to be the representation of any set $A \cap S^{1}(p, \varepsilon)$, where $\varepsilon$ satisfies the conditions of Property 1 .

A semi-algebraic set $A$ in $\mathbf{R}^{2}$ also behaves conically towards infinity. To see this, we embed $\mathbf{R}^{2}$ as the $x y$-plane in $\mathbf{R}^{3}$ and map $A$ from this embedded plane onto the sphere $S^{2}((0,0,1), 1)$, that rests on the $x y$-plane, in the direction of its north pole $(0,0,2)$. If we then add the north pole to this set as the point at infinity of the semi-algebraic set, rotate the sphere such that $(0,0,2)$ becomes the origin, and stereographically project back on the $x y$-plane, then we can look at the cone of $(0,0)$ in the resulting semi-algebraic set as the cone of the point at infinity in $A$.

This implies that for a semi-algebraic set $A$, there exists an $\varepsilon>0$ such that $\left\{(x, y) \mid x^{2}+y^{2} \geq \varepsilon^{2}\right\} \cap A$ is homeomorphic to $\{(\lambda x, \lambda y) \mid(x, y) \in$ $\left.S^{1}((0,0), \varepsilon) \cap A \wedge \lambda \geq 1\right\}$. We can indeed view the latter set as the cone with top $\infty$ and base $S^{1}((0,0), \varepsilon) \cap A$. Remark that the cone of $\infty$ in $A$ is ( ) if and only if $A$ is a bounded subset of $\mathbf{R}^{2}$.

More formally, consider the embedding $e$ of $\mathbf{R}^{2}$ in $\mathbf{R}^{3}$ that maps $(x, y)$ to $(x, y, 0)$. Let $\sigma$ be the reflection of $\mathbf{R}^{3}$ defined by $(x, y, z) \mapsto(x, y, 2-z)$. Finally, let $h: e\left(\mathbf{R}^{2}\right) \cup\{\infty\} \rightarrow S^{2}((0,0,1), 1)$ be the homeomorphism of that maps the Alexandrov one-point compactification of $e\left(\mathbf{R}^{2}\right)$ stereographically onto the sphere $S^{2}((0,0,1), 1)$, i.e., $h(x, y, 0)=\frac{4}{4+x^{2}+y^{2}}\left(x, y, \frac{x^{2}+y^{2}}{2}\right)$ and $h(\infty)=(0,0,2)$. 
Definition 2 Let $A$ be a closed semi-algebraic set in $\mathbf{R}^{2}$. We define the cone of $\infty$ in $A$ to be the cone of the point $(0,0)$ in the set $e^{-1}\left(h^{-1}(\sigma(\{(0,0,2)\} \cup\right.$ $h(e(A))) \backslash\{\infty\}))$.

We now prove that Definitions 1 and 2 are sound.

Proposition 1 Let $A$ be a closed semi-algebraic set in $\mathbf{R}^{2}$ and let $p$ be a point of $A$.

(a) The notion of the cone of $p$ in $A$ is well-defined.

(b) The notion of the cone of $\infty$ in $A$ is well-defined.

Proof. (b) The mapping $e$, the reflection $\sigma$ and the homeomorphism $h$ are semi-algebraic functions. $e^{-1}\left(h^{-1}(\sigma(\{(0,0,2)\} \cup h(e(A))) \backslash\{\infty\})\right)$ is therefore a semi-algebraic subset of $\mathbf{R}^{2}$ (see, e.g., [6]). It is also closed. This reduces the proof of Case (b) to that of Case (a).

(a) Let $p$ be a point of the closed semi-algebraic set $A$. If $p$ is an isolated point of $A$, well-definedness is trivial. Thus, assume that $p$ is not an isolated point of $A$. We have to prove that any two values $\varepsilon_{1}$ and $\varepsilon_{2}$ that satisfy the conditions of Property 1 give rise to the same finite representation. Let $\varepsilon_{1}$ and $\varepsilon_{2}$ be such values and let $h_{1}$ and $h_{2}$ be corresponding homeomorphisms of which Property 1 guarantees the existence. Assume $\varepsilon_{1}<\varepsilon_{2}$. Because of condition $(i)$ of Property $1, h_{2}\left(A \cap S^{1}\left(p, \varepsilon_{1}\right)\right)$ is the intersection of $S^{1}\left(p, \varepsilon_{1}\right)$ and the cone $h_{2}\left(A \cap B^{2}\left(p, \varepsilon_{2}\right)\right)$. The latter has $A \cap S^{1}\left(p, \varepsilon_{2}\right)$ as its base. Therefore, the homothety with center $p$ and factor $\varepsilon_{2} / \varepsilon_{1}$ maps $h_{2}\left(A \cap S^{1}\left(p, \varepsilon_{1}\right)\right)$ to $A \cap S^{1}\left(p, \varepsilon_{2}\right)$. Homothetic subsets of circles are clearly represented in the same way. Condition $(i)$ of Property 1 implies $h_{2}\left(S^{1}\left(p, \varepsilon_{1}\right)\right)=S^{1}\left(p, \varepsilon_{1}\right)$. So, $h_{2}$ induces an homeomorphism of $S^{1}\left(p, \varepsilon_{1}\right)$. To complete the proof, it suffices to show that $h_{2}$ is orientation-preserving. This follows directly from a classical result by J.W. Alexander (see, e.g., [16], page 81): A homeomorphism of $B^{2}\left(p, \varepsilon_{2}\right)$ that is the identity on $S^{1}\left(p, \varepsilon_{2}\right)$ is isotopic to the identity mapping, and therefore orientation-preserving.

Let $\mathcal{C}$ be the set of all possible cones. We define:

Definition 3 Let $A$ be a closed semi-algebraic set in $\mathbf{R}^{2}$. The point-structure of $A$ is the function $\Pi(A)$ from $A \cup\{\infty\}$ to $\mathcal{C}$ that maps each element to its cone. 
The following are topological properties of closed semi-algebraic sets.

Property $\mathbf{2}$ Let $A$ be a closed semi-algebraic set in $\mathbf{R}^{2}$.

(i) $\Pi(A)^{-1}$ is empty on all but a finite number of cones,

(ii) $\Pi(A)^{-1}$ is infinite or empty for the cones $(R),(L L)$, and $F$,

(iii) the number of points in $A$ with a cone different from $(R),(L L)$, or $F$ is finite.

Proof. The semi-algebraic sets depicted in Figure 1 have infinitely many points with cone $(R),(L L)$, and $F$. This proves part of (ii). To prove the remainder, we consider a Nash-stratification (see, e.g., Chapter 9 of [6]) $A=$ $\bigcup_{i=1}^{n} A_{i}$, where each $A_{i}$ is diffeomorphic to a point, to $] 0,1[$ or to $] 0,1\left[^{2}\right.$, and such that $A_{i} \cap \bar{A}_{j} \neq \emptyset$ for $i \neq j$ implies that $A_{i} \subset \bar{A}_{j}$ and $\operatorname{dim}\left(A_{i}\right)<\operatorname{dim}\left(A_{j}\right){ }^{4}$ All points in a 1-dimensional $A_{i}$ in this stratification therefore have

1. cone $(L L)$ (if $A_{i}$ is not adherent to a 2-dimensional stratum), or

2. cone $(R)$ (if $A_{i}$ is adherent to one 2-dimensional stratum), or

3. cone $F$ (if $A_{i}$ is adherent to two 2-dimensional strata).

Because the points in a 2-dimensional stratum also have cone $F$, removing from $A$ the points with cone $F,(L L)$ and $(R)$ results in a subset of the 0dimensional strata of $A$. There are finitely many 0-dimensional strata.

Further on, we will refer to the points with a cone different from $(R)$, $(L L)$, or $F$ as the singular points of the semi-algebraic set. Part (iii) of Property 2 shows that a closed semi-algebraic set has only a finite number of singular points. Non-singular points are also called regular points (for an illustration see Figure 3). (ii) of Property 2 shows that there are infinitely many regular points if there are any.

Definition 4 Let $A$ and $B$ be closed semi-algebraic sets in $\mathbf{R}^{2}$. We say that $\Pi(A)$ is isomorphic to $\Pi(B)$ (denoted by $\Pi(A) \cong \Pi(B))$ if there is a bijection $f$ from $A \cup\{\infty\}$ to $B \cup\{\infty\}$ with $f(\infty)=\infty$, such that $\Pi(A)=\Pi(B) \circ f$.

${ }^{4}$ The topological closure of $A$ is denoted by $\bar{A}$. The dimension of a set diffeomorphic to $] 0,1\left[^{n}\right.$ is $n$ (see [6]). 


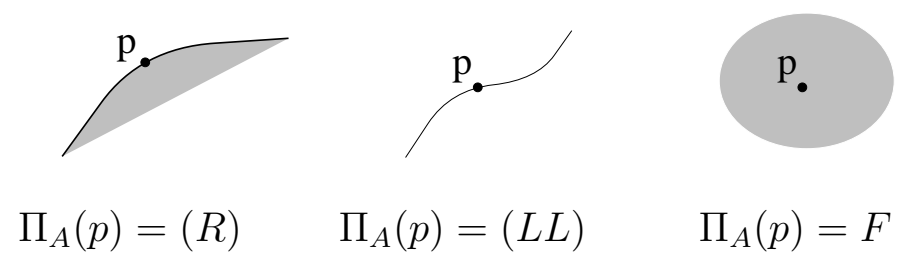

Figure 3: Regular points of a closed semi-algebraic set.

\section{The main results}

The main result of this paper is a characterization of $\mathcal{I}$-equivalence in terms of point-structure isomorphism:

Theorem 1 Let $A$ and $B$ be closed semi-algebraic sets in $\mathbf{R}^{2}$. The sets $A$ and $B$ are $\mathcal{I}$-equivalent if and only if $\Pi(A) \cong \Pi(B)$.

The proof will be given in the next section. A corollary of the theorem is a similar characterization of $\mathcal{H}$-equivalence:

Theorem 2 Let $A$ and $B$ be closed semi-algebraic sets in $\mathbf{R}^{2}$ and let $\sigma$ be some fixed reflection of $\mathbf{R}^{2}$. The sets $A$ and $B$ are $\mathcal{H}$-equivalent if and only if $\Pi(A) \cong \Pi(B)$ or $\Pi(A) \cong \Pi(\sigma(B))$.

Proof of Theorem 2. Assuming Theorem 1, we have to prove that $A$ and $B$ are $\mathcal{H}$-equivalent if and only if $A$ and $B$ are $\mathcal{I}$-equivalent or $A$ and $\sigma(B)$ are $\mathcal{I}$-equivalent. The if-implication follows from the fact that every homeomorphism of $\mathbf{R}^{2}$ is either an isotopy or isotopic to $\sigma[10,21]$.

For the only-if-implication, assume on the contrary that $A$ and $B$ are $\mathcal{H}$-equivalent and that there exist $\mathcal{I}$-invariant sentences $\varphi(S)$ and $\varphi^{\prime}(S)$ such that $A \models \varphi(S), B \not \models \varphi(S), A \models \varphi^{\prime}(S)$, and $\sigma(B) \not \models \varphi^{\prime}(S)$. Consider the sentence $\bar{\varphi}(S)$ defined by

$$
\bar{\varphi}(S)=\left(\varphi(S) \vee \varphi^{\prime}(\sigma(S))\right) \wedge\left(\varphi^{\prime}(S) \vee \varphi(\sigma(S))\right) .
$$

It can be easily shown that a sentence is $\mathcal{H}$-invariant if and only if it is invariant under $\sigma$ and it is $\mathcal{I}$-invariant. Clearly, $\bar{\varphi}(S)$ is invariant under $\sigma$. It can also be easily verified that it is $\mathcal{I}$-invariant. So, $\bar{\varphi}(S)$ is $\mathcal{H}$-invariant, and $A=\bar{\varphi}(S)$ but $B \not \models \bar{\varphi}(S)$. This contradicts the assumption. 

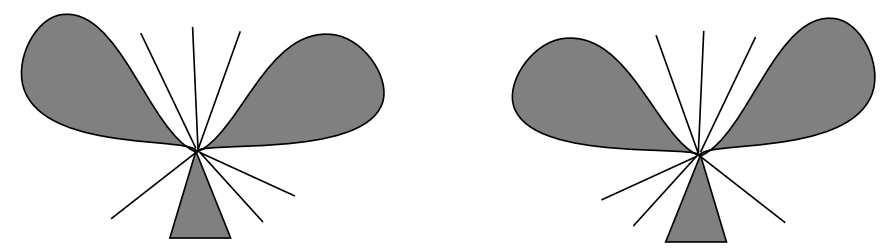

Figure 4: Two closed semi-algebraic sets that are $\mathcal{H}$-equivalent but not $\mathcal{I}$ equivalent.

Corollary 1 Topological connectivity of 2-dimensional spatial databases is not first-order expressible.

Proof. One closed disk and two separate closed disks have isomorphic pointstructures: the points on the border have $(R)$ as cone, and the points in the interior have $F$ as cone. Both sets have infinitely many of both of these types of points and both are empty towards infinity. As a consequence of Theorem 1 , they are $\mathcal{I}$-equivalent, hence also $\mathcal{H}$-equivalent.

\section{Examples.}

- Although all points on the unit circle and all points on the $x$-axis have the same cone (namely, $(L L)$ ), these semi-algebraic sets do not have isomorphic point-structures. Indeed, in the former set the cone of $\infty$ is ( ) (in other words, this semi-algebraic set is bounded), while in the latter set the cone of $\infty$ is $(L L)$.

- Figure 4 shows two semi-algebraic sets that are not $\mathcal{I}$-equivalent. Indeed, the cone of the center point in the left set is ( $L L L R L L R L R)$, while that on the right is $(L L L R L R L L R)$. The two semi-algebraic sets are of course $\mathcal{H}$-equivalent since they are mirror images of each other. We point out that it is possible that two mirror images are still $\mathcal{I}$ equivalent; for instance, the two semi-algebraic sets shown in Figure 1 have isomorphic point-structures.

In classical logic, if $A$ and $A^{\prime}$ are elementary equivalent and $B$ and $B^{\prime}$ are elementary equivalent, then the disjoint union of $A$ and $B$ and the disjoint union of $A^{\prime}$ and $B^{\prime}$ are also elementary equivalent. The following corollary shows that this property carries over to our setting only partially. 
Corollary 2 Let $A, A^{\prime}, B$ and $B^{\prime}$ be closed semi-algebraic sets in $\mathbf{R}^{2}$ such that $A \cap B=A^{\prime} \cap B^{\prime}=\emptyset$.

(i) If $A$ and $A^{\prime}$ are $\mathcal{I}$-equivalent and $B$ and $B^{\prime}$ are $\mathcal{I}$-equivalent, then $A \cup A^{\prime}$ and $B \cup B^{\prime}$ are $\mathcal{I}$-equivalent.

(ii) If $A$ and $A^{\prime}$ are $\mathcal{H}$-equivalent and $B$ and $B^{\prime}$ are $\mathcal{H}$-equivalent then $A \cup A^{\prime}$ and $B \cup B^{\prime}$ are not necessarily $\mathcal{H}$-equivalent.

Proof. Theorem 1 implies (i). For (ii), take $A, A^{\prime}$, and $B$ to be the semialgebraic set on the left of Figure 4 , and take $B^{\prime}$ to be the one on the right.

\section{The proof}

This section is devoted to the proof of Theorem 1. First, we give a number of background results on finite-model theory that will play a major role in our proofs.

\subsection{Background on finite-model theory}

In this section, we state a generic collapse result by Benedikt, Dong, Libkin, and Wong [3] and some of its implications on the first-order definability of properties of finite structures over the reals.

Consider the following decision problems on finite structures:

- The decision problem MAJORITy about two finite sets $R_{1}$ and $R_{2}$ is: $\operatorname{MAJORITY}\left(R_{1}, R_{2}\right)$ is true if and only if $R_{1} \subseteq R_{2}$ and $\left|R_{1}\right| \leq 2\left|R_{2}\right|$;

- For any set $V$ of integers, the decision problem CARD $V$ about two finite sets $R_{1}$ and $R_{2}$ is: $\mathrm{CARD}_{V}\left(R_{1}, R_{2}\right)$ is true if and only if $\left|R_{1}\right|-\left|R_{2}\right| \in V$;

- The decision problem PARITy about a finite set $R$ is: $\operatorname{PARITy}(R)$ is true if and only if $|R|$ is even.

The following lemma is a routine exercise in finite-model theory (it can, e.g., be proven using Ehrenfeucht-Fraïssé games) [11]. 
Lemma 1 On finite ordered structures in the language $\left(<, R_{1}, R_{2}\right)$, the decision problems MAJORITY $\left(R_{1}, R_{2}\right)$ and $\operatorname{CARD}_{V}\left(R_{1}, R_{2}\right)$ (where $V$ is not the empty set or the set of all integers) are not uniformly first-order expressible. On finite ordered structures in the language $(<, R)$, the decision problem $\operatorname{PARITY}(R)$ is not uniformly first-order expressible.

Benedikt, Dong, Libkin, and Wong proved that any first-order formula over the reals that is invariant under monotone bijections from $\mathbf{R}$ to $\mathbf{R}$ is equivalently expressible on finite semi-algebraic sets in the restriction of this logic that only uses order constraints. This result was a breakthrough in the line of research towards understanding of the expressive power of first-order logic over the reals and related structures $[2,4,5,13,14,17,22]$.

Consider structures in the language $\left(0,1,+, \times,<, S_{1}, \ldots, S_{k}\right)$ that are expansions of $\mathbf{R}$ with $k$ finite relations on $\mathbf{R}$. We call such structures "finite structures over the reals". A first-order formula in the language $(0,1,+, \times$, $\left.<, S_{1}, \ldots, S_{k}\right)$ is called order-generic if on such structures, it is invariant under monotone bijections $f: \mathbf{R} \rightarrow \mathbf{R}$. Benedikt, Dong, Libkin, and Wong showed the following [3]:

Theorem 3 For each order-generic first-order formula in the language (0,1, $\left.+, \times,<, S_{1}, \ldots, S_{k}\right)$, there exists a first-order formula in the language $\left(<, S_{1}\right.$, $\left.\ldots, S_{k}\right)$, that is equivalent to it on finite structures over the reals. Furthermore, in the latter formula the quantifiers may be assumed to range only over the elements actually occurring in the relations $S_{1}, \ldots, S_{k}$.

The following lemma, which specializes Lemma 1 from general finite ordered structures to finite structures over the reals, now follows directly from Theorem 3 and Lemma 1.

Lemma 2 On finite structures over the reals, the decision problems $\operatorname{PARITY}(R)$, MAJORITY $\left(R_{1}, R_{2}\right)$, and $\operatorname{CARD}_{V}\left(R_{1}, R_{2}\right)$ (where $V$ is not the empty set or the set of all integers) are not first-order expressible.

\subsection{The proof of Theorem 1}

Transformation rules. The crucial tool in the proof consists of the following three transformations rules that locally change closed semi-algebraic sets: 


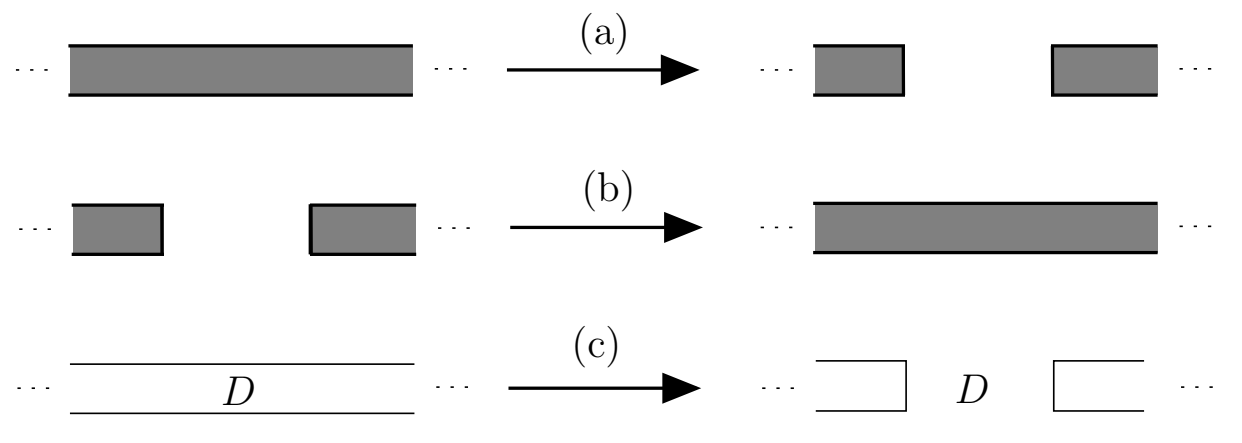

Figure 5: The transformation rules: (a) strip-cut, (b) strip-paste, (c) linecut\&paste.

Strip-cut: The strip-cut transformation, shown in Figure 5(a), locally cuts a strip in the semi-algebraic set in two.

Strip-paste: The strip-paste transformation, shown in Figure 5(b), is the inverse of strip-cut.

Line-cut\&paste: The line-cutÉpaste transformation, shown in Figure 5(c), locally cuts two lines in the semi-algebraic set and connects the corresponding loose ends. An isolated part $D$ of the semi-algebraic set may be present between the lines, which will come free after the cut\&paste.

Note that the line-cut\&paste transformation is its own inverse.

A fundamental property of the transformation rules is:

Proposition 2 Let $A$ and $B$ be closed semi-algebraic sets in $\mathbf{R}^{2}$. If $B$ is obtained from $A$ by a strip-cut, a strip-paste, or a line-cutEpaste transformation, then $A$ and $B$ are $\mathcal{I}$-equivalent.

Proposition 2 is proven in a number of steps:

1. First, a variation of Proposition 2 is proven for weak versions of the three transformation rules (Lemma 3). These weak transformations are illustrated in Figure 6. The difference between the weak strip-cut and weak strip-paste (arrows (a) and (b) in Figure 6) and the strip-cut and 


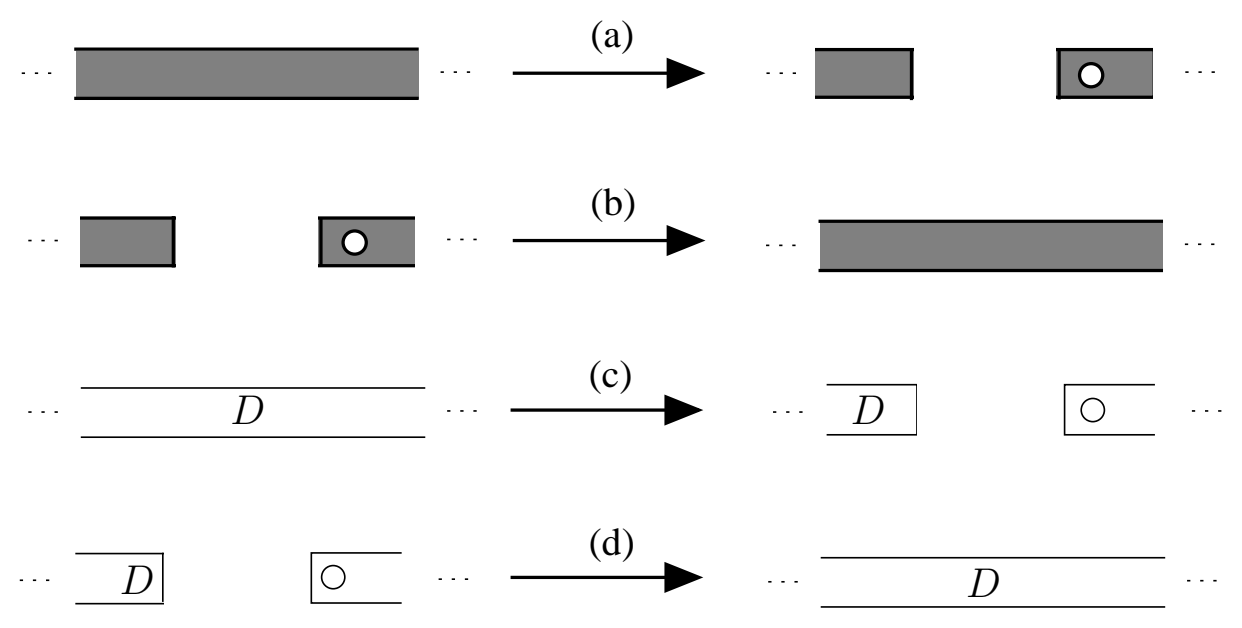

Figure 6: Weak forms of the three transformation rules: (a) weak strip-cut, (b) weak strip-paste, (c) weak line-cut, (d) weak line-paste.

strip-paste is a hole in one of the strips. Line-cut\&paste is split in a weak line-cut (arrow (c) in Figure 6) and a weak line-paste (arrow (d) in Figure 6). Here the difference is an additional circle.

2. The gap between the weak rules and the original rules is then closed via the notions of 2-regular and 1-regular semi-algebraic set (Lemmas 4 and 5).

Lemma 3 Let $A$ and $B$ be closed semi-algebraic sets in $\mathbf{R}^{2}$. If $B$ is obtained from $A$ by a weak transformation rule, then $A$ and $B$ are $\mathcal{I}$-equivalent.

Proof. We first prove the lemma for weak strip-cut and its inverse, weak strip-paste.

Assume, for the sake of contradiction, that there exist closed semi-algebraic sets $A$ and $B$ that differ by one weak strip-cut transformation but that are not $\mathcal{I}$-equivalent. So there exists an $\mathcal{I}$-invariant first-order sentence $\varphi(S)$ such that $A=\varphi(S)$ and $B \not \models \varphi(S)$. Consider the decision problem MAJORITY about two finite sets of reals $R_{1}$ and $R_{2}$ (see Section 5.1). We will prove the existence of a formula $\psi_{A}\left(x, y, R_{1}, R_{2}\right)$ in the language of the reals with 


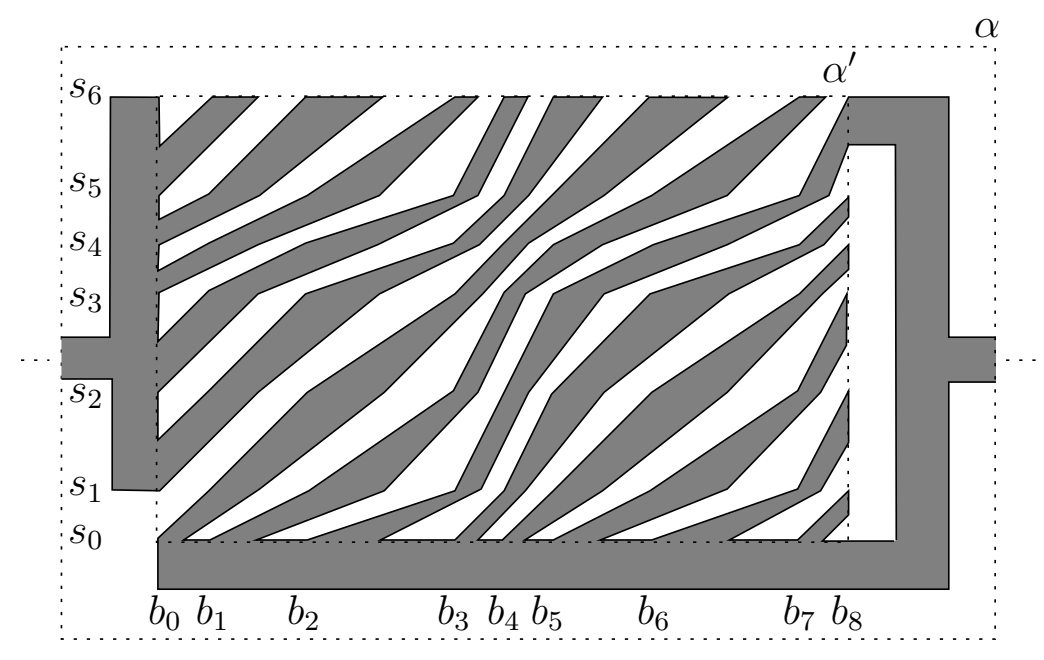

Figure 7: Construction of $D\left(R_{1}, R_{2}\right)$ for $R_{1}=\{1,3,5,6,7,9\}$ and $R_{2}=$ $\{1,3,6,7\}$ in the rectangular area $\alpha$.

two unary relation symbols, $R_{1}$ and $R_{2}$, and two free variables, $x$ and $y$, that defines a subset $D_{A}\left(R_{1}, R_{2}\right)=\left\{(x, y) \mid \psi_{A}\left(x, y, R_{1}, R_{2}\right)\right\}$ of $\mathbf{R}^{2}$ such that $D_{A}\left(R_{1}, R_{2}\right) \models \varphi(S)$ if and only if MAJority $\left(R_{1}, R_{2}\right)$ is false. By Lemma 2 , this then yields the desired contradiction. The reduction technique we thus use is inspired by work of Grumbach and $\mathrm{Su}$ [13].

Obviously, the part $R_{1} \subseteq R_{2}$ can be tested in first-order logic. For given $R_{1}=\left\{r_{1}, \ldots, r_{n}\right\}$ and $R_{2}=\left\{a_{1}, \ldots, a_{m}\right\}$ with $0<r_{1}<\cdots<r_{n}$ and $0<a_{1}<\cdots<a_{m}$, we construct within the fixed rectangular part $\alpha$ of $\mathbf{R}^{2}$, where the weak strip-cut takes place, a closed semi-algebraic set $D\left(R_{1}, R_{2}\right)$ consisting of interconnected strips.

This construction is similar to constructions by Grumbach and $\mathrm{Su}$ (in [13]) and is illustrated in Figure 7 for $n=6$ and $m=4$. The construction is as follows. Take a rectangular subarea $\alpha^{\prime}$ of $\alpha$. Let $\left(b_{0}, s_{0}\right)$ be the left bottom corner of $\alpha^{\prime}$ and let $h$ and $w$ be its height and width. Then sets $R_{1}^{\prime}=$ $\left\{s_{0}, \ldots, s_{n}\right\}$ and $R_{2}^{\prime}=\left\{b_{0}, b_{1}, \ldots, b_{m}, b_{m+1}, \ldots, b_{2 m}\right\}$, with $s_{i}=s_{0}+r_{i} h / r_{n}$ 
$(0<i \leq n), b_{i}=b_{0}+a_{i} w / 2 a_{m}$ and $b_{m+i}=b_{i}+w / 2(0<i \leq m)$ are constructed. Then, the following closed strips of $D\left(R_{1}, R_{2}\right)$ are constructed:

1. the filled convex quadrangle with corners $\left(b_{i}, s_{j}\right),\left(\left(b_{i}+b_{i+1}\right) / 2, s_{j}\right)$, $\left(b_{i+1}, s_{j+1}\right),\left(\left(b_{i+1}+b_{i+2}\right) / 2, s_{j+1}\right)$ for $0<i<2 m-1$ and $0 \leq j<n$ and for $i=j=0$,

2. the filled convex quadrangle with corners $\left(b_{2 m-1}, s_{j}\right),\left(\left(b_{2 m-1}+b_{2 m}\right) / 2, s_{j}\right)$, $\left(b_{2 m}, s_{j+1}\right),\left(b_{2 m},\left(s_{j}+s_{j+1}\right) / 2\right)$ for $0 \leq j<n$,

3. the filled convex quadrangle with corners $\left(b_{0},\left(s_{j+1}+s_{j+2}\right) / 2\right),\left(\left(b_{1}+\right.\right.$ $\left.\left.b_{2}\right) / 2, s_{j+2}\right),\left(b_{1}, s_{j+2}\right),\left(b_{0}, s_{j+1}\right)$ for $0 \leq j<n-1$.

Finally, a number of additional closed strips are added in the area $\alpha \backslash \alpha^{\prime}$ (as illustrated in Figure 7) to complete the construction of $D\left(R_{1}, R_{2}\right)$. Remark that the complete construction of $D\left(R_{1}, R_{2}\right)$, as described above, starting from $R_{1}$ and $R_{2}$ can be expressed by a formula in the language of the reals with two unary relation symbols to represent the sets $R_{1}$ and $R_{2}$.

We then glue $D\left(R_{1}, R_{2}\right)$ to the part of $A$ outside the strip-cut area $\alpha$. In this part $A$ and $B$ are identical. We thus obtain a semi-algebraic set $D_{A}\left(R_{1}, R_{2}\right)$, which can be described by a formula over $R_{1}$ and $R_{2}$. The construction is such that $D\left(R_{1}, R_{2}\right)$ is isotopic to the right part of Figure 6(a) if $\operatorname{MAJORITy}\left(R_{1}, R_{2}\right)$ is true, and isotopic to the left part of Figure 6(a) otherwise. Hence, in case of majority $D_{A}\left(R_{1}, R_{2}\right)$ is isotopic to $B$, and in the other case it is isotopic to $A$. Since $\varphi(S)$ is $\mathcal{I}$-invariant and distinguishes between $A$ and $B$, can use $\varphi(S)$ to express MAJORITY.

For the weak line-cut and the weak line-paste the lemma can be proven with the same technique. The border of $D\left(R_{1}, R_{2}\right)$ is used, rather then $D\left(R_{1}, R_{2}\right)$ itself.

Definition 5 A bounded semi-algebraic set in $\mathbf{R}^{2}$ is called 2-regular if it is not empty and all of its points have either $F$ or $(R)$ as cone. A bounded semi-algebraic set in $\mathbf{R}^{2}$ is called 1-regular if it is not empty and all of its points have $(L L)$ as cone.

Lemma 4 Let $A$ and $B$ be closed semi-algebraic sets in $\mathbf{R}^{2}$ and let $O$ be an open disk in $\mathbf{R}^{2}$. 

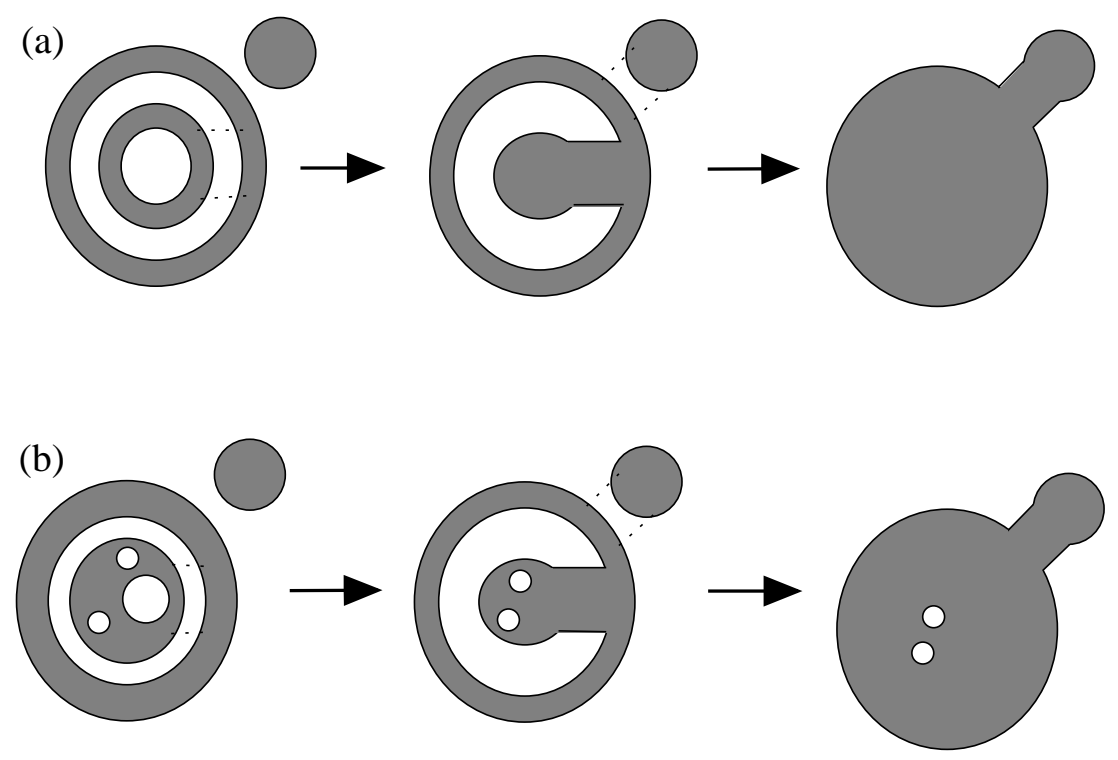

Figure 8: Transformation of (a) a set with $\gamma=1$ to a disk, and (b) a set with $\gamma=-1$ to a disk with two holes.

1. If $A \cap O$ is 2-regular, then replacing $A \cap O$ in $O$ by any other 2-regular semi-algebraic set, yields a semi-algebraic set that is $\mathcal{I}$-equivalent to $A$.

2. If $A \cap O$ is 1-regular, then replacing $A \cap O$ in $O$ by any other 1-regular semi-algebraic set, yields a semi-algebraic set that is $\mathcal{I}$-equivalent to $A$.

Proof. We only give the proof for the 2-regular case; the 1-regular case is analogous.

For any 2-regular semi-algebraic set $B$, define $\gamma(B)$ to be the number of connected components of $B$ minus the number of holes in $B$. It can be easily shown that $B$ can be transformed by a finite number of applications of weak strip-cut and weak strip-paste either to the disjoint union of $\gamma(B)$ disks, if $\gamma(B)>0$, or to one disk with $1-\gamma(B)$ holes, if $\gamma(B) \leq 0$. This is illustrated in Figure 8 (a) for $\gamma=1$ and in Figure 8 (b) for $\gamma=-1$.

Since weak strip-cut and weak strip-paste preserve $\gamma$, the lemma thus follows from Lemma 3, when we restrict attention to replacing $A \cap O$ by a set with the same value for $\gamma$. 
Now suppose, for the sake of contradiction, there are 2-regular semialgebraic sets $B_{1}$ and $B_{2}$ with different values for $\gamma$ for which the lemma does not hold. In other words, there exists an $\mathcal{I}$-invariant sentence $\varphi(S)$ such that $A\left(B_{1}\right) \models \varphi(S)$ and $A\left(B_{2}\right) \not \models \varphi(S)$, where $A(B)$ denotes the semialgebraic set obtained from $A$ by replacing $A \cap O$ by $B$. Define the following subset of $\mathbf{Z}$ (the integers):

$$
Z_{\varphi}^{A}=\{\gamma(B) \mid(\mathbf{R}, A(B)) \models \varphi(S)\} .
$$

If we can show that $Z_{\varphi}^{A}=\emptyset$ or $Z_{\varphi}^{A}=\mathbf{Z}$, this will contradict the assumption that $\gamma\left(B_{1}\right) \in Z_{\varphi}^{A}$ and $\gamma\left(B_{2}\right) \notin Z_{\varphi}^{A}$ and complete the proof.

Thereto, for any set $V$ of integers, consider the decision problem $\mathrm{CARD}_{V}$ about two finite sets of reals $R_{1}$ and $R_{2}$ (see Section 5.1). From Lemma 2 it follows that if $\mathrm{CARD}_{V}$ is first-order expressible in the language $(0,1,+$, $\left.\times,<, R_{1}, R_{2}\right)$, then $V=\emptyset$ or $V=\mathbf{Z}$. Hence, to complete the proof of this lemma, it remains to show that $\mathrm{CARD}_{Z_{\varphi}^{A}}$ is so expressible. This can be proven by a reduction (similar to the one of the proof of Lemma 3) of $\mathrm{CARD}_{Z_{\varphi}^{A}}$ to $\varphi(S)$. Given $R_{1}=\left\{r_{0}, r_{1}, \ldots, r_{n}\right\}$ and $R_{2}=\left\{s_{0}, s_{1}, \ldots, s_{m}\right\}$ (without loss of generality we can assume that $r_{0}<r_{1}<\cdots<r_{n}, s_{0}<s_{1}<\cdots<s_{m}$, and that the points $\left(s_{0}-1, r_{0}-1\right)$ and $\left(s_{m}+1, r_{n}+1\right)$ are located in the disk $O$ ), we construct within $O$ a 2-regular semi-algebraic set $D\left(R_{1}, R_{2}\right)$ as follows (this construction is illustrated in Figure 9 for $R_{1}=\{0,1,2,3,4,5\}$ and $\left.R_{2}=\{0,1,2,3,4\}\right)$.

Within the rectangle with left bottom corner $\left(s_{0}, r_{0}\right)$ and right upper corner $\left(s_{m}, r_{n}\right)$ (the dotted rectangle $\alpha$ in Figure 9$)$ the set $D\left(R_{1}, R_{2}\right)$ consists of the the following filled quadrangles:

1. the filled convex quadrangle with corners $\left(s_{i}, r_{j}\right),\left(\left(s_{i}+s_{i+1}\right) / 2, r_{j}\right)$, $\left(s_{i+1}, r_{j+1}\right),\left(\left(s_{i+1}+s_{i+2}\right) / 2, r_{j+1}\right)$ for $0<i<m-1$ and $0 \leq j<n$ and for $i=j=0$,

2. the filled convex quadrangle with corners $\left(s_{m-1}, r_{j}\right),\left(\left(s_{m-1}+s_{m}\right) / 2, r_{j}\right)$, $\left(s_{m}, r_{j+1}\right),\left(s_{m},\left(r_{j}+r_{j+1}\right) / 2\right)$ for $0 \leq j<n$,

3. the filled convex quadrangle with corners $\left(s_{0},\left(r_{j+1}+r_{j+2}\right) / 2\right),\left(\left(s_{1}+\right.\right.$ $\left.\left.s_{2}\right) / 2, r_{j+2}\right),\left(s_{1}, r_{j+2}\right),\left(s_{0}, r_{j+1}\right)$ for $0 \leq j<n-1$.

Then, two filled horizontal rectangular strips $\left(\left\{(x, y) \mid\left(r_{0}-1 / 2 \leq y \leq\right.\right.\right.$ $\left.\left.\left.r_{0} \vee r_{n} \leq y \leq r_{n}+1 / 2\right) \wedge s_{0} \leq x \leq s_{m}+1 / 2\right\}\right)$ and one filled vertical 


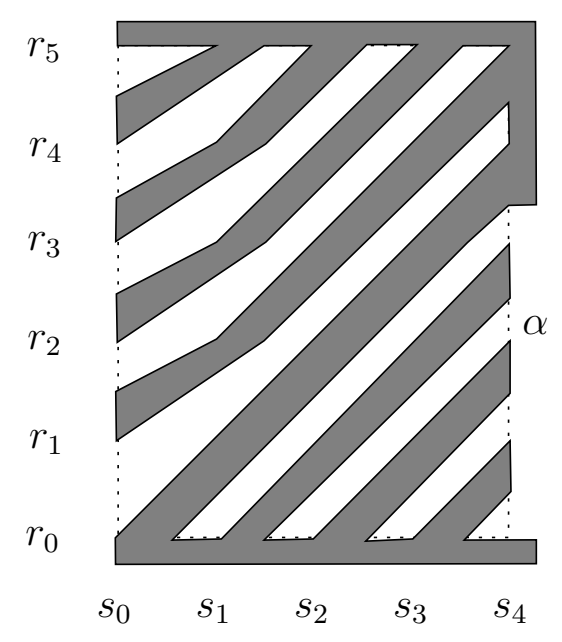

Figure 9: Construction of $D\left(R_{1}, R_{2}\right)$ in $O$ for $R_{1}=\{0,1,2,3,4,5\}$ and $R_{2}=$ $\{0,1,2,3,4\}$.

rectangular strip $\left(\left\{(x, y) \mid\left(r_{n-2}+r_{n-1}\right) / 2 \leq y \leq r_{n} \wedge s_{m} \leq x \leq s_{m}+1 / 2\right\}\right)$ are added to $D\left(R_{1}, R_{2}\right)$ outside $\alpha$ but inside the rectangle with left bottom corner $\left(s_{0}-1, r_{0}-1\right)$ and right upper corner $\left(s_{m}+1, r_{n}+1\right)$, as shown in Figure 9 .

The closed semi-algebraic set $A\left(D\left(R_{1}, R_{2}\right)\right)$ can be described by a formula over $R_{1}$ and $R_{2}$. The construction is such that $\gamma\left(D\left(R_{1}, R_{2}\right)\right)=\left|R_{1}\right|-\left|R_{2}\right|$. Hence, since we already know that $A\left(D\left(R_{1}, R_{2}\right)\right)$ is $\mathcal{I}$-equivalent to $A(B)$ for any other 2-regular set $B$ with $\gamma(B)=\left|R_{1}\right|-\left|R_{2}\right|$, we can use $\varphi(S)$ on $A\left(D\left(R_{1}, R_{2}\right)\right)$ to decide $\mathrm{CARD}_{Z_{\varphi}^{A}}\left(R_{1}, R_{2}\right)$.

Lemma 5 Let $A$ be a closed semi-algebraic set and $O$ be an open disk in $\mathbf{R}^{2}$. If $A \cap O$ consists of a part $D$ surrounded by a circle, then replacing $A \cap O$ in $O$ by a circle and $D$ outside this circle, yields a set that is $\mathcal{I}$-equivalent to $A$.

Proof. Suppose, for the sake of contradiction, that there is a set $A$ violating the lemma. Let $A^{\prime}$ be the set obtained from $A$ by moving $D$ outside the circle. Denote this circle by $C$. Since $A$ violates the lemma there is an $\mathcal{I}$-invariant sentence $\varphi(S)$ such that $A \models \varphi(S)$ and $A^{\prime} \not \neq \varphi(S)$. 


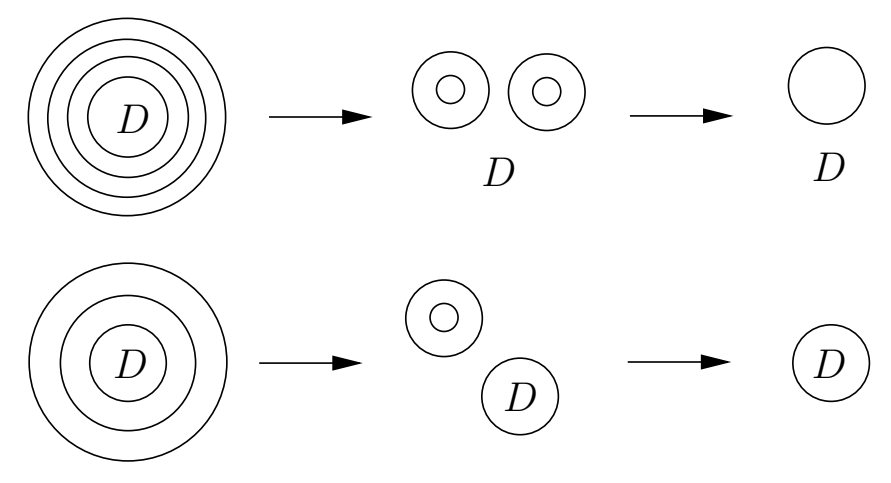

Figure 10: Illustration of the proof of Lemma 5.

Consider the decision problem PARITY about a finite set of reals $R$ (see Section 5.1). We will prove the existence of a formula $\psi_{A}(x, y, R)$ in the language of the reals with one unary relation symbol, $R$, and two free variables, $x$ and $y$, that defines a subset $D_{A}(R)=\left\{(x, y) \mid \psi_{A}\left(x, y, R_{1}, R_{2}\right)\right\}$ of $\mathbf{R}^{2}$ such that $D_{A}(R) \models \varphi(S)$ if and only if $\operatorname{PARITY}(R)$ is false. This yields the desired contradiction, since Lemma 2 says that PARITY is not first-order expressible in this language. The construction here is quite different from the ones used in the proofs of Lemmas 3 and 4. It is illustrated in Figure 10.

Let $R=\left\{r_{1}, \ldots, r_{n}\right\}$, with $1 / 4=r_{1}<\cdots<r_{n}<1$ (without loss of generality). Let $p$ be a point in $O$ and let $\varepsilon$ be such that $B^{2}(p, \varepsilon) \subset O$. We construct a closed semi-algebraic set $D_{A}(R)$ which is the disjoint union of the following parts:

- for each $i=1, \ldots, n$, the circle with center $p$ and radius $r_{i} / \varepsilon$;

- a semi-algebraic isotopic deformation of $A$ such that $D$ fits in the inner circle and the part of $A$ outside $O$ is outside $B^{2}(p, \varepsilon)$, from which we remove the image of the circle $C$.

This semi-algebraic set can be described by a first-order formula over $R$. Obviously, within the disk $B^{2}(p, \varepsilon)$ in $D_{A}(R), D$ is surrounded by $n$ circles.

By going from the outside to the inside, we can repeatedly cut pairs of circles using the weak line-cut transformation. Every cut produces a pair of nested circles. By an isotopy we can bring these nested circles into one open disk $O^{\prime}$ in $\mathbf{R}^{2}$. We distinguish two possibilities: 


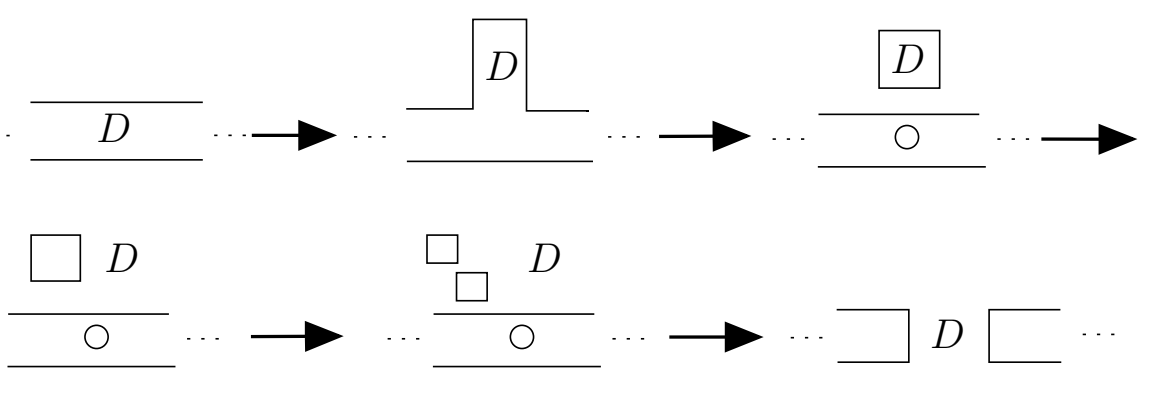

Figure 11: The proof of the line-cut\&paste transformation.

- If $n$ is even, this cutting process eventually leaves $D$ unsurrounded. By Lemma 4 we can replace the nested circles in $O^{\prime}$ by a single circle and we obtain a semi-algebraic set isotopic to $A^{\prime}$.

- If $n$ is odd, this leaves $D$ surrounded by a single circle. We now replace the nested circles in $O^{\prime}$ by one single pair of nested circles, and apply one final weak line-paste operation to obtain a semi-algebraic set isotopic to $A$.

We have thus shown that if $n$ is even, $D_{A}(R)$ is $\mathcal{I}$-equivalent to $A^{\prime}$, and if $n$ is odd, $D_{A}(R)$ is $\mathcal{I}$-equivalent to $A$. Hence, $\varphi(S)$ can be used on $D_{A}(R)$ to decide $\operatorname{PARITY}(R)$.

We can now give the

Proof of Proposition 2. We only give the proof for the line-cut\&paste transformation. The proof for the strip-cut and the strip-paste transformation is similar. The proof is illustrated in Figure 11. First, the semi-algebraic set is isotopically deformed. The weak line-cut\&past is applied (second arrow). Then Lemma 5 is applied (third arrow). Next, Lemma 4 is applied (fourth arrow). The last arrow in the figure comprises three applications of weak line-cut\&paste.

The transformation process. Having our tools, as furnished by Proposition 2 , in place, we now show: 
Proposition 3 Let $A$ and $B$ be closed semi-algebraic sets in $\mathbf{R}^{2}$ such that $\Pi(A) \cong \Pi(B)$. Then $A$ and $B$ can be transformed, by a finite sequence of strip-cut, strip-paste and line-cut\&paste transformations and isotopies, into one and the same semi-algebraic set.

Proof. Since $\Pi(A) \cong \Pi(B)$, there is a bijection $f$ from $A \cup\{\infty\}$ to $B \cup\{\infty\}$ that maps points to points with the same cone. In particular, $f$ maps the singular points in $A$ to the singular points in $B$ with matching cones.

Let $\operatorname{Sing}(A)$ be the set of singular points of $A$ together with the point at infinity, $\infty$. For each $p$ in $\operatorname{Sing}(A)$ and its corresponding point $f(p)$ in $\operatorname{Sing}(B)$ we proceed as follows. From each $R$, i.e., each region, in the cone of $p$ we cut out a bounded lobe coming out of $p$, using the strip-cut transformation. If at least one $L$ appears in the cone of $p$, we choose one such $L$ in the cone of $p$, i.e. a line $l$ ending in $p$, and then use line-cut\&paste to connect $l$ and the next line (in the clockwise order around $p$ ) into a loop starting and ending in $p$. We continue this process in the clockwise order around $p$ until all lines, or all lines but one (in case their number is odd), form loops. We hereby make sure that no isolated parts of the semi-algebraic set become trapped in these loops. We perform the same transformations around $f(p)$ in $B$, starting from a line $l^{\prime}$ that corresponds to the same $L$ in the cone of $f(p)$ in $B$. If the number of lines in the point $p$, and thus in $f(p)$, is even, we obtain a "flower" around $p$ and $f(p)$. If the number is odd, we obtain a "flower with a stem". This stem is necessarily connected to another flower.

As "residual material" of the process we get isolated, bounded, regular parts. This material can be transformed to a single closed disk, a single circle, or the disjoint union of a closed disk and a circle, depending on which cones appear in the residue. If a flower with points of type $(L L)$ is present, the circle can even be absorbed by this flower, and similarly for the disk. In case no $L$ appears in the cone of all singular points, the circle cannot be absorbed. In this case, we can make sure that no part of the semi-algebraic set is located in the interior of the circle (using Lemma 5).

After this process, all connected components are situated in the same area of $\mathbf{R}^{2}$.

The only way in which the resulting semi-algebraic sets can still differ is that stems can connect different flowers. We can interchange stems by isotopically bringing them into a parallel position (this is possible since all 
stems are in the same area) and by then using a line-cut\&paste transformation. This finally yields isotopic semi-algebraic sets.

An illustration of the transformation process is given in Figure 12.

We are finally ready for:

Proof of Theorem 1. The if-implication $(\Pi(A) \cong \Pi(B)$ implies that $A$ and $B$ are $\mathcal{I}$-equivalent) is immediate from Propositions 2 and 3.

For the only-if implication, assume $\Pi(A) \approx \Pi(B)$. Then there exists at least one cone for which $A$ has a different number of points than $B$, or the cone of $\infty$ in $A$ is different from the cone of $\infty$ in $B$. It is therefore sufficient to show that there exists a first-order sentence that expresses that a closed semi-algebraic set has exactly $n$ points having some fixed cone $c$, and that there exists a sentence that expresses that the cone of $\infty$ is some fixed cone $c$. Since the cone of a point in a semi-algebraic set is invariant under isotopies (this can be shown using the techniques used in the proof of Proposition 1), such a sentence is certainly $\mathcal{I}$-invariant. It clearly suffices to show that there exists a formula $\psi_{c}(x, y, S)$ that expresses that the point $(x, y)$ has cone $c$ in $S$ and that there exists a sentence $\psi_{c}(S)$ that expresses that $\infty$ has cone $c$ in $S$.

For points in $\mathbf{R}^{2}$, if $c=F$, the wanted sentence is $(\exists \varepsilon \neq 0)\left(\forall x^{\prime}\right)\left(\forall y^{\prime}\right)\left(\left(x^{\prime}-\right.\right.$ $\left.x)^{2}+\left(y^{\prime}-y\right)^{2}<\varepsilon^{2} \rightarrow S\left(x^{\prime}, y^{\prime}\right)\right)$, and if $c=()$, this sentence is $(\exists \varepsilon \neq$ $0)\left(\forall x^{\prime}\right)\left(\forall y^{\prime}\right)\left(0<\left(x^{\prime}-x\right)^{2}+\left(y^{\prime}-y\right)^{2}<\varepsilon^{2} \rightarrow \neg S\left(x^{\prime}, y^{\prime}\right)\right)$. For $\infty$, there are similar sentences.

For other cones, the definition of the desired formula is based on the following topological property of semi-algebraic sets in $\mathbf{R}^{2}$. This property is a consequence of the property that semi-algebraic sets are locally conical around each point, including $\infty$. The proof of the lemma follows directly from Property 1 and the proof of Proposition 1.

Lemma 6 Let $p$ be a point of a closed semi-algebraic set in $\mathbf{R}^{2}$. There exists an $\varepsilon_{0}>0$ such that for every $\varepsilon$, with $0<\varepsilon<\varepsilon_{0}$, the cone with top $p$ and base $S^{1}(p, \varepsilon) \cap A$ is isotopic to the one with base $S^{1}\left(p, \varepsilon_{0}\right) \cap A$. There also exists an $\varepsilon_{0}>0$ such that for every $\varepsilon$, with $\varepsilon>\varepsilon_{0}$, the cone with top $(0,0)$ and base $S^{1}((0,0), \varepsilon) \cap A$ is isotopic to the one with base $S^{1}\left((0,0), \varepsilon_{0}\right) \cap A$.

By this lemma, it is sufficient to show that there is a sentence $\psi_{c}^{\prime}(x, y, \varepsilon, S)$ that expresses that the circle with radius $\varepsilon$ and center $(x, y)$ has an intersection with the semi-algebraic set that corresponds to the cone $c$. The desired 

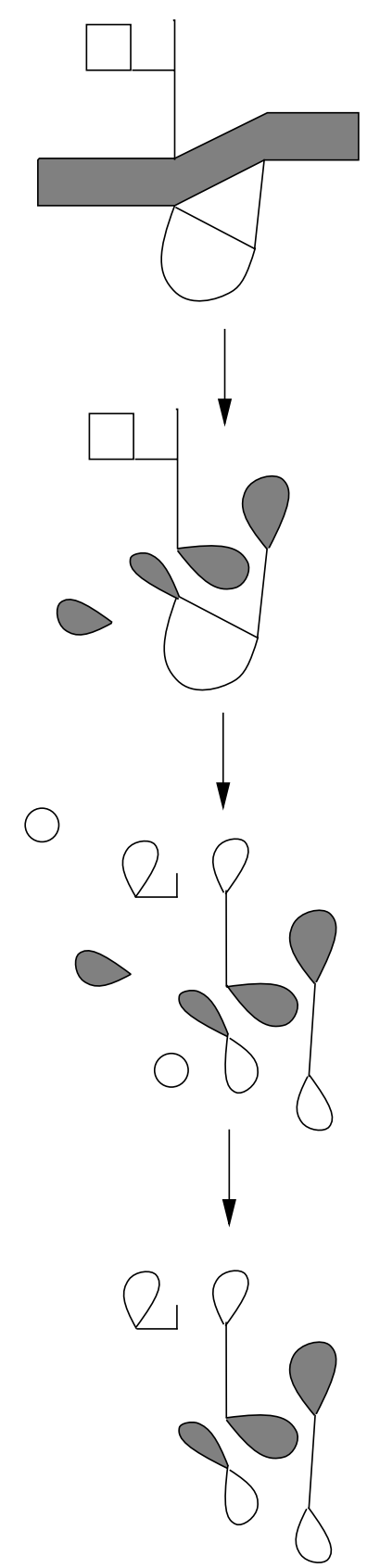
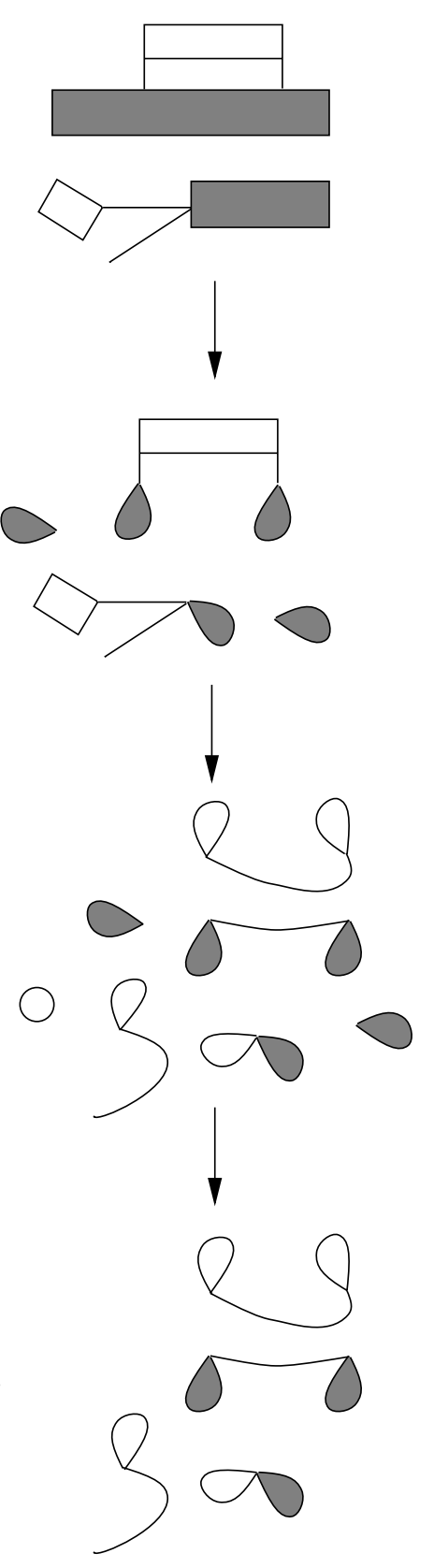

Figure 12: Two semi-algebraic sets with isomorphic point-structures and their transformation into the same set. After the first step, lobes have been cut out. After the second step, loops have been formed. In the third step, stems are interchanged in the bottom-right set to obtain a semi-algebraic set isotopic to the bottom-left one. 


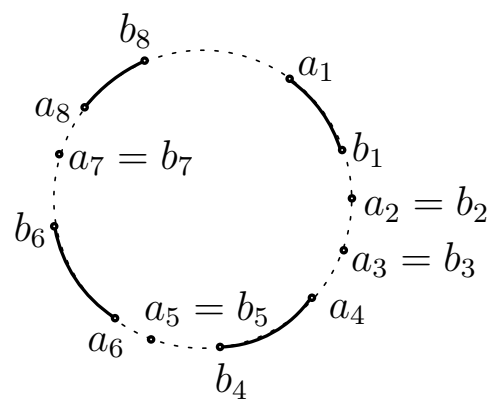

Figure 13: The intersection of a closed semi-algebraic set with a circle.

formula is then, as a consequence of the previous lemma, $\left(\exists \varepsilon_{0}\right)(\forall \varepsilon)(0<\varepsilon \leq$ $\left.\varepsilon_{0} \rightarrow \psi_{c}^{\prime}(x, y, \varepsilon, S)\right)$ for a point $(x, y)$ and $\left(\exists \varepsilon_{0}\right)(\forall \varepsilon)\left(\varepsilon>\varepsilon_{0} \rightarrow \psi_{c}^{\prime}(0,0, \varepsilon, S)\right)$ for $\infty$.

If the intersection is not empty or the complete circle it is a finite union of points and closed arc segments, as already explained in Section 3. Such an intersection is illustrated in Figure 13. The intersection corresponds to a non-empty circular list $\left(\alpha_{1} \cdots \alpha_{n}\right)$ with $\alpha_{i} \in\{L, R\}$. The case $\alpha_{i}=R$ corresponds to an arc-segment on the circle that is completely determined by its end points $a_{i}$ and $b_{i}$ ( $b_{i}$ comes after $a_{i}$ in a clockwise sense). If $\alpha_{i}=L$, then $a_{i}=b_{i}$ is a single point on the circle.

The following sentence then describes the intersection of the set $S$, with the circle $S^{1}((x, y), \varepsilon)$ up to an isotopy of $S^{1}((x, y), \varepsilon)$ :

$$
\begin{gathered}
\left(\exists a_{1 x}\right)\left(\exists a_{1 y}\right)\left(\exists b_{1 x}\right)\left(\exists b_{1 y}\right) \cdots\left(\exists a_{n x}\right)\left(\exists a_{n y}\right)\left(\exists b_{n x}\right)\left(\exists b_{n y}\right) \\
\left(( \forall x ^ { \prime } ) ( \forall y ^ { \prime } ) \left(\left(x^{\prime}-x\right)^{2}+\left(y^{\prime}-y\right)^{2}=\varepsilon^{2} \rightarrow\right.\right. \\
\left(\left(\left(\bigvee_{i=1}^{n} \operatorname{Between}_{(x, y), \varepsilon}\left(x^{\prime}, y^{\prime}, a_{i x}, a_{i y}, b_{i x}, b_{i y}\right)\right) \leftrightarrow S\left(x^{\prime}, y^{\prime}\right)\right) \wedge\right. \\
\left(\bigvee_{i=1}^{n} \operatorname{Between}_{(x, y), \varepsilon}\left(x^{\prime}, y^{\prime}, b_{i x}, b_{i y}, a_{((i+1) \bmod n) x}, a_{((i+1) \bmod n) y}\right)\right) \\
\left.\left.\left.\left.\leftrightarrow \neg S\left(x^{\prime}, y^{\prime}\right)\right)\right)\right)\right),
\end{gathered}
$$

where $\operatorname{Between}_{(x, y), \varepsilon}\left(x^{\prime}, y^{\prime}, x_{1}, y_{1}, x_{2}, y_{2}\right)$ abbreviates the formula that expresses for points $\left(x^{\prime}, y^{\prime}\right),\left(x_{1}, y_{1}\right)$ and $\left(x_{2}, y_{2}\right)$ on $S^{1}((x, y), \varepsilon)$ that $\left(x^{\prime}, y^{\prime}\right)$ is equal to $\left(x_{1}, y_{1}\right)$ or to $\left(x_{2}, y_{2}\right)$ or is located between the clockwise ordered pair of points $\left(\left(x_{1}, y_{1}\right),\left(x_{2}, y_{2}\right)\right)$ of $S^{1}((x, y), \varepsilon)$. The formula $\operatorname{Between}_{(x, y), \varepsilon}\left(x^{\prime}, y^{\prime}\right.$, $\left.x_{1}, y_{1}, x_{2}, y_{2}\right)$ can be written as a disjunction over all possible positions of 
$\left(x_{1}, y_{1}\right)$ and $\left(x_{2}, y_{2}\right)$ in the four quadrants of $S^{1}((x, y), \varepsilon)$.

The disjunct for the case where both $\left(x_{1}, y_{1}\right)$ and $\left(x_{2}, y_{2}\right)$ are in the first quadrant of $S^{1}((x, y), \varepsilon)$ is

$$
\begin{gathered}
\left(x \leq x_{1} \leq x^{\prime} \leq x_{2} \wedge y \leq y_{2} \leq y^{\prime} \leq y_{1}\right) \vee \\
\left(x \leq x_{2} \leq x_{1} \leq x^{\prime} \wedge y \leq y^{\prime} \leq y_{1} \leq y_{2}\right) \vee \\
\left(x \leq x^{\prime} \leq x_{2} \leq x_{1} \wedge y \leq y_{1} \leq y_{2} \leq y^{\prime}\right)
\end{gathered}
$$

In this formula, the first disjunct takes care of the case where $\left(x_{2}, y_{2}\right)$ comes after $\left(x_{1}, y_{1}\right)$ in the first quadrant (in the clockwise sense). The two other disjuncts cover the other case.

This completes the proof.

\section{Two corollaries}

Theorems 1 and 2 have two interesting corollaries. The first one is the following:

Theorem $4 \mathcal{I}$ - and $\mathcal{H}$-equivalence are decidable properties of closed semialgebraic sets in $\mathbf{R}^{2}$.

Proof. Theorems 1 and 2 show that a decision algorithm for $\mathcal{I}$-equivalence implies a decision algorithm for $\mathcal{H}$-equivalence.

A decision algorithm for $\mathcal{I}$-equivalence is as follows:

$V_{A}:=\emptyset ; V_{B}:=\emptyset ;$

for each cone c do

$A_{\mathbf{c}}:=\{p \in A \cup\{\infty\} \mid \Pi(A)(p)=\mathbf{c}\} ;$

$B_{\mathbf{c}}:=\{p \in B \cup\{\infty\} \mid \Pi(B)(p)=\mathbf{c}\}$;

if $\neg\left(\left|A_{\mathbf{c}}\right|=\left|B_{\mathbf{c}}\right| \wedge\left(\infty \in A_{\mathbf{c}} \leftrightarrow \infty \in B_{\mathbf{c}}\right)\right)$ then return false;

$V_{A}:=V_{A} \cup A_{\mathbf{c}} ; V_{B}:=V_{B} \cup B_{\mathbf{c}}$;

if $V_{A}=A \cup\{\infty\} \wedge V_{B}=B \cup\{\infty\}$ then return true

od.

The algorithm tests whether $\Pi(A) \cong \Pi(B)$.

It should first be noted that all possible cones can be effectively enumerated, e.g., by starting with the cone $F$, and then enumerating all circular 
lists over the set $\{L, R\}$ in some order. The sets $A_{\mathbf{c}}$ and $B_{\mathbf{c}}$ can be first-order defined over the semi-algebraic sets $A$ and $B$ (see the only-if direction in the proof of Theorem 1) and hence are themselves semi-algebraic.

The test $\left|A_{\mathbf{c}}\right|=\left|B_{\mathbf{c}}\right|$ can be performed as follows. Either $\mathbf{c}$ is $F,(R)$, or $(L L)$, in which case the test amounts to testing that $A_{\mathbf{c}}$ and $B_{\mathbf{c}}$ are both empty or both not empty. This is a test obviously expressible in first-order logic and therefore decidable for semi-algebraic sets by Tarski's theorem [23]. If $\mathbf{c}$ is another kind of cone, then both $A_{\mathbf{c}}$ and $B_{\mathbf{c}}$ are finite and symbolic algorithms for the first-order theory of the reals $[1,7,19,8]$ can effectively enumerate them. The for-loop always terminates since in each closed semialgebraic set only a finite number of cones can appear (see (i) of Property 2).

In order to formulate the second corollary, we call two semi-algebraic sets $\mathcal{I}$-equivalent under $<$ if they cannot be distinguished by a $\mathcal{I}$-invariant sentence in the restricted language $(<, S)$ (i.e., not using the symbols $0,1,+$ and $\times)$.

We have the following result:

Theorem 5 Two closed semi-algebraic sets in $\mathbf{R}^{2}$ are $\mathcal{I}$-equivalent if and only if they are $\mathcal{I}$-equivalent under $<$. The same holds for $\mathcal{H}$-equivalence.

To prove this corollary we need an analogue of Lemma 6 in terms of rectangles instead of circles. This analogue is not straightforward since the number of degrees of freedom is higher in the case of rectangles. Let Rect $t_{x, y, x^{\prime}, y^{\prime}}$ denote the rectangle that has $(x, y)$ and $\left(x^{\prime}, y^{\prime}\right)$ as diagonally opposite corner points.

Lemma 7 Let $A$ be a closed semi-algebraic set in $\mathbf{R}^{2}$.

(a) Let $p$ be a point with coordinates $\left(x_{p}, y_{p}\right)$ of $A$. There exist $x_{0}, y_{0}, x_{1}, y_{1}$ such that $x_{0}<x_{p}<x_{1}$ and $y_{0}<y_{p}<y_{1}$ and such that for every $x_{0}^{\prime}, y_{0}^{\prime}, x_{1}^{\prime}, y_{1}^{\prime}$ with $x_{0}<x_{0}^{\prime}<x_{p}<x_{1}^{\prime}<x_{1}$ and $y_{0}<y_{0}^{\prime}<y_{p}<y_{1}^{\prime}<y_{1}$, the cone with base $\operatorname{Rect}_{x_{0}, y_{0}, x_{1}, y_{1}} \cap A$ and top $p$ is isotopic to that with base $\operatorname{Rect}_{x_{0}^{\prime}, y_{0}^{\prime}, x_{1}^{\prime}, y_{1}^{\prime}} \cap A$.

(b) There also exist $x_{0}, y_{0}, x_{1}, y_{1}$ such that $x_{0}<x_{1}$ and $y_{0}<y_{1}$ and such that for every $x_{0}^{\prime}, y_{0}^{\prime}, x_{1}^{\prime}, y_{1}^{\prime}$ with $x_{0}^{\prime}<x_{0}<x_{1}<x_{1}^{\prime}$ and $y_{0}^{\prime}<y_{0}<y_{1}<$ $y_{1}^{\prime}$ the cone with base $\operatorname{Rect}_{x_{0}, y_{0}, x_{1}, y_{1}} \cap A$ and top the origin is isotopic to the one with base $\operatorname{Rect}_{x_{0}^{\prime}, y_{0}^{\prime}, x_{1}^{\prime}, y_{1}^{\prime}} \cap A$. 
Proof of Lemma 7. Let $A$ be a closed semi-algebraic set in $\mathbf{R}^{2}$. The desired rectangles will be derived from a refinement of Collins's Cylindrical Algebraic Decomposition (CAD) of $\mathbf{R}^{2}$ with respect to $A$. Collins proves that, given some first-order definition of $A$, there exists a $\mathrm{CAD} \mathcal{C}$ of $\mathbf{R}^{2}$ such that each cell in $\mathcal{C}$ entirely belongs to $A$ or to the complement of $A[1,7,8]$.

More technically, Collins shows that given a quantifier-free formula $\varphi(x, y)$ over the reals that describes $A$ (i.e., $\left.A=\left\{(x, y) \mid(x, y) \in \mathbf{R}^{2} \wedge \varphi(x, y)\right\}\right)$, there exists a partition $\mathcal{C}_{x}=\left(S_{1}, S_{2}, \ldots, S_{2 \nu+1}\right)$ of the $x$-axis of $\mathbf{R}^{2}$, where either $\nu=0$ and $S_{1}=\mathbf{R}$, or $\nu>0$ and there exist real algebraic numbers $\alpha_{1}<\alpha_{2}<\cdots<\alpha_{\nu}$ such that $S_{2 i}=\left\{\alpha_{i}\right\}$ for $1 \leq i \leq \nu, S_{2 i+1}$ is the open interval $\left(\alpha_{i}, \alpha_{i+1}\right)$ for $1 \leq i<\nu, S_{1}=\left(-\infty, \alpha_{1}\right)$, and $S_{2 \nu+1}=\left(\alpha_{\nu}, \infty\right)$ and there exists a partition $\mathcal{C}=\left(S_{1,1}, \ldots, S_{1,2 \nu_{1}+1}, \ldots, S_{\nu, 1}, \ldots, S_{2 \nu+1,2 \nu_{2 \nu+1}+1}\right)$ of $\mathbf{R}^{2}$ (each $S_{i, j}$ is called a cell of the CAD) such that for $1 \leq i \leq 2 \nu+1$, there exist continuous real-valued algebraic functions $f_{i, 1}<f_{i, 2}<\cdots<f_{i, \nu_{i}}$ on $S_{i}$ such that if $\nu_{i}=0, S_{i, 1}=S_{i} \times \mathbf{R}$, if $\nu_{i}>0, S_{i, 2 j}=\left\{(x, y) \mid x \in S_{i} \wedge y=\right.$ $\left.f_{i, j}(x)\right\}$ for $1 \leq j \leq \nu_{i}, S_{i, 2 j+1}=\left\{(x, y) \mid x \in S_{i} \wedge f_{i, j}(x)<y<f_{i, j+1}(x)\right\}$ for $1 \leq j<\nu_{i}, S_{i, 1}=\left\{(x, y) \mid x \in S_{i} \wedge y<f_{i, 1}(x)\right\}$, and $S_{i, 2 \nu_{i}+1}=\{(x, y) \mid$ $\left.x \in S_{i} \wedge f_{i, \nu_{i}}(x)<y\right\}$ for $1 \leq j<\nu_{i}$, and such that all polynomials $p(x, y)$ appearing in the quantifier-free formula $\varphi(x, y)$ that describes $A$, are sign invariant (i.e., $>0,<0$, or $=0$ ) on each of the cells of $\mathcal{C}$. Therefore, each of the cells of $\mathcal{C}$ entirely belongs to $A$ or to the complement of $A$, and $A$ is the disjoint union of a finite number of cells of $\mathcal{C}$.

We can refine this $\mathrm{CAD}$ by first refining $\mathcal{C}_{x}$ in the $x$-coordinates of all extremal points and points of inflection of the functions $f_{i, j}$, and then refining the $\mathrm{CAD} \mathcal{C}$ by building the appropriate stacks on these new singleton elements of $\mathcal{C}_{x}$ and on the split intervals of $\mathcal{C}_{x}$. We can therefore assume that the functions $f_{i, j}$ are constant, (strictly) monotone concave, or (strictly) monotone convex functions of $x$ on the intervals of $\mathcal{C}_{x}$.

As an illustration, in Figure 14, the twenty five cells $c_{1}, \ldots, c_{25}$ of $\mathcal{C}$ for the semi-algebraic set $\left\{(x, y) \mid x^{2}+y^{2} \leq 1 \vee(y=0 \wedge x \geq 1)\right\}$ are indicated. The partition $\mathcal{C}_{x}$ consists of seven elements. The cells of a CAD of $A$ are either 0-dimensional, 1-dimensional, or 2-dimensional (defined in the obvious way). The cells $c_{3}, c_{11}, c_{13}$ and $c_{21}$ are the 0-dimensional cells of $\mathcal{C}$ of Figure 14 . Of these only the cell $c_{21}$ contains a singular point of the set. The cells $c_{6}, c_{8}, c_{16}, c_{18}$, and $c_{24}$ are the 1-dimensional cells and the remaining cells are 2-dimensional. 


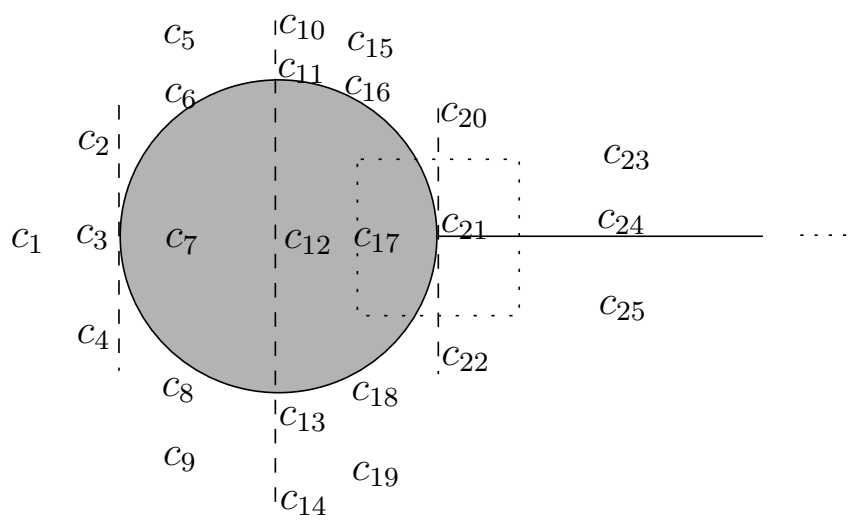

Figure 14: An example of a CAD of $\mathbf{R}^{2}$ for the set described by the formula $x^{2}+y^{2} \leq 1 \vee(y=0 \wedge x \geq 1)$.

We now proceed to the prove of (a). We show how the values of $x_{0}$, $x_{1}, y_{0}$, and $y_{1}$ can be chosen, given the $\mathrm{CAD} \mathcal{C}$ of $A$, for a singular point $p=\left(x_{p}, y_{p}\right)$ of $A$. For regular points of $A$ the proof is similar. The singular point $p$ belongs to the stack of $\mathcal{C}$ that is built on $\left\{x_{p}\right\} \in \mathcal{C}_{x}$. Let $d_{0}$ be the distance between $x_{p}$ and the next smaller single point cell in $\mathcal{C}_{x}$, if such element of $\mathcal{C}_{x}$ exists, or else let $d_{0}$ be 1 . Similarly, let $d_{1}$ be the distance between $x_{p}$ and the next larger single point cell in $\mathcal{C}_{x}$ if such element of $\mathcal{C}_{x}$ exists or else let $d_{1}$ be 1 . Take $x_{0}=x_{p}-d_{0} / 2$ and $x_{1}=x_{p}+d_{1} / 2$. Let $e_{0}$ be the distance in the $y$-direction between $p$ and the 0 -dimensional cell of $\mathcal{C}$ with next smaller $y$-coordinate if such a cell exists or let $e_{0}$ be 1 otherwise. Let $e_{1}$ be the distance in the $y$-direction between $p$ and the 0 -dimensional cell of $\mathcal{C}$ with next larger $y$-coordinate or 1 if such a cell does not exist. Take $y_{0}=y_{p}-e_{0} / 2$ and $y_{1}=y_{p}+e_{1} / 2$.

In Figure 14, Rect $_{x_{0}, y_{0}, x_{1}, y_{1}}$ is shown in dashed lines for $\{p\}=c_{21}$.

For all $x_{0}^{\prime}, y_{0}^{\prime}, x_{1}^{\prime}, y_{1}^{\prime}$ with $x_{0} \leq x_{0}^{\prime}<x_{p}<x_{1}^{\prime} \leq x_{1}$ and $y_{0} \leq y_{0}^{\prime}<$ $y_{p}<y_{1}^{\prime} \leq y_{1}$, the interior of the rectangle Rect $_{x_{0}^{\prime}, y_{0}^{\prime}, x_{1}^{\prime}, y_{1}^{\prime}}$ contains no other 0 -dimensional cell of $\mathcal{C}$ except for the cell $\{p\}$. Every vertical 1-dimensional cell with endpoint in $p$ intersects Rect $_{x_{0}^{\prime}, y_{0}^{\prime}, x_{1}^{\prime}, y_{1}^{\prime}}$ exactly once. For every nonvertical 1-dimensional cell the same is true because these cells are the graphs of constant or monotonic concave or convex functions $f_{i, j}$ on the open in- 
tervals of $\mathcal{C}_{x}$ that start or end in $x_{p}$. The 2-dimensional cells of $\mathcal{C}$ that are adherent to $p$ are wrapped between 1-dimensional cells that arrive in $p$. Let $p_{1}, \ldots, p_{k}$ be the intersection points of $\operatorname{Rect}_{x_{0}^{\prime}, y_{0}^{\prime}, x_{1}^{\prime}, y_{1}^{\prime}}$ with the 1-dimensional cells of $\mathcal{C}$ (given in clockwise order). Let $q_{1}, \ldots, q_{k}$ be the corresponding intersection points of the 1-dimensional cells with Rect $_{x_{0}, y_{0}, x_{1}, y_{1}}$. It is now clear that there exists an isotopy $h$ of $\mathbf{R}^{2}$ that maps the line segment $p p_{i}$ to the line segment $p q_{i}$ for $i=1, \ldots, k$ and that maps the rectangle segment $p_{i} p_{(i+1) \bmod k}$ of Rect $\operatorname{Rx}_{x_{0}^{\prime}, y_{0}^{\prime}, x_{1}^{\prime}, y_{1}^{\prime}}$ to the segment $q_{i} q_{(i+1) \bmod k}$ of $\operatorname{Rect}_{x_{0}, y_{0}, x_{1}, y_{1}}$ for $i=1, \ldots, k$. The isotopy $h$ therefore maps the cone with top $p$ and base Rect $_{x_{0}^{\prime}, y_{0}^{\prime}, x_{1}^{\prime}, y_{1}^{\prime}} \cap A$ to the one with top $p$ and base $\operatorname{Rect}_{x_{0}, y_{0}, x_{1}, y_{1}} \cap A$.

To prove (b), we show how the values of $x_{0}, x_{1}, y_{0}$, and $y_{1}$ can be chosen, given the $\mathrm{CAD} \mathcal{C}$ of $A$. Let $d_{0}$ and $d_{1}$ be 0 if $\nu=0$ (i.e., if $\mathcal{C}_{x}=\left\{S_{1}\right\}$ ) and $d_{0}$ be $\alpha_{1}$ and $d_{1}$ be $\alpha_{\nu}$ if $\nu>0$ (the elements $S_{2}$ and $S_{2 \nu}$ of $\mathcal{C}_{x}$ are respectively $\left\{\alpha_{1}\right\}$ and $\left\{\alpha_{\nu}\right\}$ ). Let $e_{0}$ and $e_{1}$ be 0 if $\mathcal{C}$ has no 0 -dimensional cell, else let $e_{0}$ be the $y$-coordinate of the 0 -dimensional cell of $\mathcal{C}$ with minimal $y$-coordinate and let $e_{1}$ be the $y$-coordinate of the 0 -dimensional cell of $\mathcal{C}$ with maximal $y$-coordinate. Take $x_{0}=d_{0}-1, x_{1}=d_{1}+1, y_{0}=e_{0}-1$ and $y_{1}=e_{1}+1$. With these values of $x_{0}, x_{1}, y_{0}$, and $y_{1}$ a similar reasoning as in Case (a) can be made to complete the proof for Case (b).

Proof of Theorem 5. The only-if implication is trivial. For the if-implication, assume $A$ and $B$ are not $\mathcal{I}$-equivalent. Then $\Pi_{A}$ and $\Pi_{B}$ are not isomorphic, and thus there exists at least one cone for which $A$ has a different number of points than $B$ or the cone of $\infty$ in $A$ is different from the cone of $\infty$ in $B$. We follow the same argumentation as in in the proof of Theorem 1 : we show that there is a sentence in the language $(<, S)$ that expresses that a point $p$ has a certain cone $c$ in $S$, and that there exists a sentence that expresses that the cone of $\infty$ in $S$ is $c$.

The intersections of the rectangles $\operatorname{Rect}_{x_{0}^{\prime}, y_{0}^{\prime}, x_{1}^{\prime}, y_{1}^{\prime}}$ around $p=\left(x_{p}, y_{p}\right)$ (or around $\infty$ ) with $A$, as they appear in Lemma 7 , are empty, the complete rectangle, or they consist of a finite number of closed rectangle segments and points. Clearly, the two former cases correspond to the empty and the full cone respectively. We will now show that, for the third case, a description of this intersection by means of a circular list of $R$ 's (for closed rectangle segments) and $L$ 's (for points) following one clockwise turn exactly coincides with the cone of $p($ or $\infty)$ as defined in Section 3 . 
Indeed, let $h$ by an isotopy of $\mathbf{R}^{2}$ that maps all small enough squares with center $p$ to circles with center $h(p)$. For $h(p)$ in $h(A)$ there exists a $\varepsilon_{0}>0$ satisfying the conditions of Property 1 . For each $\varepsilon$ with $0<\varepsilon<\varepsilon_{0}$, the description of $h^{-1}\left(S^{1}(h(p), \varepsilon)\right) \cap A$ in terms of $R$ 's and $L$ 's is the cone of $h(p)$ in $h(A)$. Since cones are preserved by isotopies, it also the cone of $p$ in $A$. From Lemma 7, it follows that therefore the description of every Rect $_{x_{0}^{\prime}, y_{0}^{\prime}, x_{1}^{\prime}, y_{1}^{\prime}} \cap A$ is the cone of $p$.

To deal with the point at infinity, let $h$ by an isotopy of $\mathbf{R}^{2}$ that maps all large enough squares with center $(0,0)$ to circles with center $h((0,0))=(0,0)$. For the origin in $h(A)$ there exists a $\varepsilon_{0}>0$ satisfying the conditions of Property 1. For each $\varepsilon$ with $\varepsilon_{0}<\varepsilon$, the description of $h^{-1}\left(S^{1}(h((0,0)), \varepsilon)\right) \cap A$ in terms of $R$ 's and $L$ 's is the cone of $\infty$ in $h(A)$. Since cones are preserved by isotopies, it also the cone of $\infty$ in $A$. From Lemma 7 , it follows that therefore the description of every $\operatorname{Rect}_{x_{0}^{\prime}, y_{0}^{\prime}, x_{1}^{\prime}, y_{1}^{\prime}} \cap A$ is the cone of $\infty$.

To complete the proof it therefore is sufficient to show that there is a sentence $\psi_{c}\left(x, y, x^{\prime}, y^{\prime}, S\right)$ over $(<, S)$ that expresses that Rect $_{x, y, x^{\prime}, y^{\prime}}$ has an intersection with $S$ that corresponds to the cone $c$. Indeed, the sentence

$$
\begin{gathered}
\left(\exists x_{0}\right)\left(\exists x_{1}\right)\left(\exists y_{0}\right)\left(\exists y_{1}\right)\left(\forall x_{0}^{\prime}\right)\left(\forall x_{1}^{\prime}\right)\left(\forall y_{0}^{\prime}\right)\left(\forall y_{1}^{\prime}\right) \\
\left(x_{0}<x_{0}^{\prime}<x_{p}<x_{1}^{\prime}<x_{1} \wedge y_{0}<y_{0}^{\prime}<y_{p}<y_{1}^{\prime}<y_{1} \rightarrow \psi_{c}\left(x_{0}^{\prime}, y_{0}^{\prime}, x_{1}^{\prime}, y_{1}^{\prime}, S\right)\right)
\end{gathered}
$$

then expresses that the point $p$ has cone $c$ in $S$, and the sentence

$$
\begin{gathered}
\left(\exists x_{0}\right)\left(\exists x_{1}\right)\left(\exists y_{0}\right)\left(\exists y_{1}\right)\left(\forall x_{0}^{\prime}\right)\left(\forall x_{1}^{\prime}\right)\left(\forall y_{0}^{\prime}\right)\left(\forall y_{1}^{\prime}\right) \\
\left(x_{0}^{\prime}<x_{0}<x_{1}<x_{1}^{\prime} \wedge y_{0}^{\prime}<y_{0}<y_{1}<y_{1}^{\prime} \rightarrow \psi_{c}\left(x_{0}^{\prime}, y_{0}^{\prime}, x_{1}^{\prime}, y_{1}^{\prime}, S\right)\right)
\end{gathered}
$$

then expresses that the cone of $\infty$ in $S$ is $c$.

Clearly, the intersection of a closed semi-algebraic set $S$ with a line segment parallel to the $x$ - or $y$-axis can be described by a sentence in the restricted language $(<, S)$. The desired sentence $\psi_{c}\left(x, y, x^{\prime}, y^{\prime}, S\right)$ is a conjunction of four such sentences.

\section{Concluding remarks}

In this paper, we have focused on closed semi-algebraic sets. However, the notion of point-structure, fundamental to our development, can also be defined for general semi-algebraic sets in $\mathbf{R}^{2}$. Unfortunately, due to the possible presence of components of the interior of semi-algebraic sets with mixed borders 
(open and closed), our transformation-based proof (in particular Proposition 3) does not carry over to this more general setting in a straightforward way.

We are also looking at other dimensions. In dimension one, the notions of $\mathcal{I}$-equivalence and $\mathcal{H}$-equivalence coincide with isotopic and homeomorphic. Generalizations to higher dimensions seem feasible. Indeed, the local cone structure around points in a semi-algebraic set, which provided the main inspiration for our work, also holds there.

\section{References}

[1] D.S. Arnon. Geometric reasoning with logic and algebra. Artificial Intelligence, 37, pages 37-60, 1988.

[2] O.V. Belegradek, A.P. Stolboushkin, and M.A. Taitslin. On ordergeneric queries. Technical Report 96-01, DIMACS, 1996.

[3] M. Benedikt, G. Dong, L. Libkin, and L. Wong. Relational expressive power of constraint query languages. In Proceedings 15th ACM Symposium on Principles of Database Systems, pages 5-16. ACM Press, 1996.

[4] M. Benedikt and L. Libkin. On the structure of queries in constraint query languages. In Proceedings of the 11th IEEE Symposium on Logic in Computer Science, pages 25-34. IEEE Computer Society Press, 1996.

[5] M. Benedikt and L. Libkin. Languages for relational databases over interpreted structures. In Proceedings of the 16th ACM Symposium on Principles of Database Systems, pages 87-98. ACM Press, 1997.

[6] J. Bochnak, M. Coste, and M.-F. Roy. Géométrie Algébrique Réelle. Springer-Verlag, 1987.

[7] B.F. Caviness, J.R. Johnson, editors. Quantifier Elimination and Cylindrical Algebraic Decomposition. Springer, Wien-New York, 1998.

[8] G.E. Collins. Quantifier elimination for real closed fields by cylindrical algebraic decomposition. In volume 33 of Lecture Notes in Computer Science, pages 134-183. Springer-Verlag, 1975. 
[9] M. Coste. Ensembles semi-algébriques. In Géometrie Algébrique Réelle et Formes Quadratiques, volume 959 of Lecture Notes in Mathematics, pages 109-138. Springer-Verlag, 1982.

[10] R.H. Cromwell and R.H. Fox. Introduction to Knot Theory, volume 57 of Graduate Texts in Mathematics. Springer-Verlag, 1977.

[11] H.D. Ebbinghaus, J. Flum. Finite Model Theory, Springer-Verlag, 1995.

[12] J. Flum and M. Ziegler. Topological Model Theory, volume 769 of Lecture Notes in Mathematics. Springer-Verlag, 1980.

[13] S. Grumbach and J. Su. Queries with arithmetical constraints. Theoretical computer Science, 173(1), pages 151-181, February 1997.

[14] S. Grumbach, J. Su, and C. Tollu. Linear constraint query languages: expressive power and complexity. In D. Leivant, editor, Logic and Computational Complexity, volume 960 of Lecture Notes in Computer Science, pages 426-446. Springer, 1995.

[15] C.W. Henson, C.G. Jockusch, Jr., L.A. Rubel, and G. Takeuti. First Order Topology, volume CXLIII of Dissertationes Mathematicae. 1977.

[16] E.E. Moise. Geometric Topology in Dimensions 2 and 3, volume 47 of Graduate Texts in Mathematics. Springer-Verlag, 1977.

[17] J. Paredaens, J. Van den Bussche, and D. Van Gucht. First-order queries on finite structures over the reals. In Proceedings of the 10th IEEE Symposium on Logic in Computer Science, pages 79-89. IEEE Computer Society Press, 1995.

[18] A. Pillay. First order topological structures and theories. Journal of Symbolic Logic, 52(3), pages 763-778, September 1987.

[19] J. Renegar. On the computational complexity and geometry of the firstorder theory of the reals. Journal of Symbolic Computation, 13, pages 255-352, 1989.

[20] A. Robinson. A note on topological model theory. Fundamenta Mathematicae, 81, pages 159-171, 1974. 
[21] J. Stillwell. Classical Topology and Combinatorial Group Theory, volume 72 of Graduate Texts in Mathematics. Springer-Verlag, 1980.

[22] A.P. Stolboushkin and M.A. Taitslin. Linear vs. order constraints over rational databases. In Proceedings of the 15th ACM Symposium on Principles of Database Systems, pages 17-27. ACM Press, 1996.

[23] A. Tarski. A Decision Method for Elementary Algebra and Geometry. University of California Press, 1951. 